\title{
Technisch generalisierte Kommunikationsmedien. Kommunikation im Medium der Adresslisten
}

\author{
Jörg Räwel, unabhängiger Wissenschaftler, Zürich \\ joerg.raewel@gmail.com
}

\begin{abstract}
Zusammenfassung
Aus systemtheoretischer Perspektive wird die Form adresslistenbasierter Kommunikation, wie sie das Internet ermöglicht - oft unter "soziale Medien» bzw. "soziale Netzwerke» kategorisiert -, untersucht. Kommunikation also etwa in Singlebörsen, sozialen Medien wie Facebook, Netzwerken beruflicher Ausrichtung, Reisekontaktbörsen, usw. Es wird gezeigt, wie Adresslisten als Medien eine technisch unterstützte Möglichkeit darstellen, das Problem doppelter Kontingenz, im Sinne von Initiative und Stabilisierung von Interaktionssystemen, zu bewältigen. Am Fallbeispiel der Anbahnung von (romantischer) Liebe, also in Bezug auf Singlebörsen, werden die Vorteile (Hoffnungen), wie auch die Nachteile (Enttäuschungen) von Adresslistenkommunikation erörtert. So wird nachgewiesen, dass hier zwar einerseits das Problem der Initiative bewältigt werden kann, aber Adresslistenkommunikation anderseits Probleme für die Kontinuität bzw. Stabilität von Interaktionssystemen innerhalb des symbolisch generalisierten Kommunikationsmediums Liebe induziert. Nicht zuletzt wird gezeigt, dass durch Adresslisten reputationsorientierte Kommunikation an Bedeutung gewinnt.
\end{abstract}

\section{Schlüsselwörter}

Soziale Medien, Soziale Netzwerke, Doppelte Kontingenz, Adresslisten, Liebe, Reputation

\begin{abstract}
From a system-theory perspective, the form of address list based communication, as made possible by the Internet - often categorized under "social media" or "social networks" - is analyzed. Examples of address-list based communication are online dating services, online social networking sites such as Facebook, networking sites for the business community, travel networking sites, etc. The paper theorizes how address-lists as media represent a technologically supported possibility to cope with the problem of double contingency in terms of initiative and stabilization of interaction systems. The paper addresses, as a case example, the advantages (hopefulness) and the disadvantages (disappointments) of address-list based communication in the initiation of love or romantic relationships. Thus, it shows that the problem of initiative can be overcome, but address list communication creates problems for the continuity or stability of interaction systems within love as a symbolically generalized medium of communication. Last, but not least, it shows that address lists increase the importance of reputation-oriented communication.
\end{abstract}

\section{Keywords}

social media, social networks, double contingency, address lists, love, reputation 


\section{Einleitung}

In vorliegendem Aufsatz werden Form und Folgen von digitaler, adresslistenbasierter Kommunikation, wie sie das Internet ermöglicht, untersucht. Kommunikation also unter Ausnutzung von (oft auch zu kaufender) Mitgliedschaft in öffentlich zugänglichen Adresslisten. ${ }^{1}$

Von Interesse ist vorliegende Untersuchung, weil digitale Adresslistenkommunikation, etwa mit Blick auf Dienste, wie Facebook, Twitter, Parship, Instagram usw., in den letzten Jahren einen enormen Aufschwung erlebt hat. Es kann also mit erheblichen gesellschaftlichen Auswirkungen gerechnet werden. Unmittelbarer Weise offenkundig im Hinblick auf die Konstituierung von Interaktionssystemen. ${ }^{2}$ Des Weiteren hat die Form möglicher Kommunikation erheblichen Einfluss auf die Form gesellschaftlicher Differenzierung. So erlaubte erst die Erfindung von Schrift gesellschaftliche Stratifikation von segmentierten Gesellschaften, die sich zuvor über Zehntausende von Jahren nur über mündliche Kommunikation reproduzierten. Allerdings kann angesichts der Neuartigkeit der internetbasierten Formen von Kommunikation nur darüber spekuliert werden, mit welchen gesellschaftlichen Veränderungen $\mathrm{zu}$ rechnen ist (etwa systemtheoretisch informiert: Baecker, 2007a).

Die Untersuchung knüpft zunächst an die grundlegende Beobachtung an, dass die Funktion uneingeschränkt aller digitalen, öffentlich zugänglichen Adresslisten vom Vorhandensein einer möglichst grossen Anzahl von Nutzerprofilen als

1 Also Singlebörsen, wie Parship, eDarling, meet2cheat, usw. (siehe Wikipedia Artikel «Singlebörse» für einen Überblick an Anbietern), Reisekontaktlisten, wie z.B. joinmytrip, Netzwerke beruflicher Ausrichtung, wie LinkedIn oder XING, Netzwerke zur Pflege gemeinsamer Interessen, wie Pinterest, Fotosharing Plattformen, wie Instagram, soziale Medien, wie Facebook und Twitter usw.

2 So haben sich mittlerweile ein Drittel aller zwischen 2005 und 2012 zustande gekommenen Partner von Ehen in den USA über das Internet kennengelernt (vgl. Cacioppo et al., 2013). gleichartigen Elementen - und damit von einem Medium - abhängt. Als Adresslisten werden hier Listen von in digitaler Form vorliegenden gleichartigen Nutzerprofilen auf der Basis elektronischer Formulare verstanden. Die digitale, gleichartige Form der Nutzerprofile ermöglicht vielfältige Formen der Datenverarbeitung. Etwa den instantanen Austausch von Information, bzw. Nachrichten zwischen Profilen über das Internet, den allzeit möglichen Zugriff auf Nutzerprofile, ein jederzeit mögliches Sperren von Nutzerprofilen, die nahezu zeitlich unbegrenzte Speicherung von Nachrichten, Anonymität, Statistiken, die für Kommunikationspräferenzen nutzbar sind, bzw. diese abbilden, usw.

Es liegt damit auf der Hand, Form Funktion und Strukturen - adresslistenbasierter Kommunikation mit Hilfe der Unterscheidung Medium/Form zu untersuchen. Vorliegende Studie erfolgt entsprechend mit den Bordmitteln der Luhmann'schen Systemtheorie (zentral Luhmann, 1984, 1997). Das Kommunikationsmodell der Systemtheorie zu Grunde gelegt, wird versucht, sich Adresslistenkommunikation theoretisch mit Hilfe der Unterscheidung Medium/Form (Luhmann, 1997, S. 190 ff.) anzunähern.

Bislang erfolgten Studien zu Adresslistenkommunikation (bzw. zu «sozialen Medien» oder «sozialen Netzwerken») theoretisch mit Hilfe des Netzwerkbegriffs, bzw. durch Netzwerkanalyse (grundlegend dazu White, 1992; in systemtheoretischer Ausrichtung vgl. Baecker, 2007b, 2012; Fuhse, 2009; im Weiteren etwa Trier, Bobrik \& Bartels, 2007, Lee \& Wellman., 2012, Frank-Job et al., 2013). In Studien derart, steht die Untersuchung der Formung des Mediums, eben zu Netzwerken, im Vordergrund. Also die kommunikative Konstruktion von Identitäten als "Knotenpunkten» von Netzwerken und deren Sozialbeziehungen als «Kanten» (vgl. Fuhse, 2009, S. $302 \mathrm{ff}$.).

Allerdings lässt diese theoretische Annäherung an Adresslistenkommunikation deren medialen Charakter unterbelichtet. Charakteristisch für digitale soziale Medien ist nicht nur die leichte Koppelung ihrer Elemente (also der Nutzerprofile), 
und so die Formung von Identitäten (Personen, Adressen, Akteuren) und Sozialbeziehungen $\mathrm{zu}$ Netzwerken, sondern ebenso deren leichte Entkoppelung. Wir gehen davon aus, dass gerade die anfänglich leicht mögliche Auflösung von so initiierten Sozialbeziehungen - also deren zunächst wiederum unkompliziert möglicher Verfall in die mediale Abstraktheit des Kommunikationsmediums - zu den charakteristischen, funktional dienlichen Eigenschaften internetbasierter sozialer Medien gehört. Es ist insbesondere dieser Aspekt von digitalen sozialen Medien, der bislang unberücksichtigt geblieben ist.

Vorliegende Studie versteht sich als eine theoriegeleitete Exploration. Es soll untersucht werden, was in den Blick gerät, wenn Adresslisten mit einer bislang unerprobten Unterscheidung (Medium/Form) beobachtet werden. Aufgrund dieses theoretisch neuen Zugangs - gewissermassen ein Biss in einen anderen Apfel (Luhmann, 1984, S. 9) - geht es zunächst nicht darum, kurzschlüssig Defizite oder Unzulänglichkeiten von Netzwerkanalysen nachzuweisen. Allenfalls mag zukünftige Forschung erweisen, dass die unterschiedlichen Ansätze auf je unterschiedlich fruchtbare Weise Wissen generieren. Ganz so, wie die systemtheoretischen Fundamentalunterscheidungen System/Umwelt und Medium/Form nicht als konkurrierende, sondern als ergänzende wissens- bzw. informationsgenerierende Unterscheidungen zu verstehen sind (vgl. Huber, 1991, S. 121). Dabei erlaubt die Neuheit der Anwendung der Unterscheidung Medium/ Form auf Adresslistenkommunikation, ganz im Sinne einer theoretischen Exploration, spekulative, gleichwohl theoriegeleitete Beobachtungen. Diese mögen zukünftige, auch empirische Forschungen inspirieren.

Der Aufsatz gliedert sich in drei Abschnitte. Im ersten Teil (I.) wird es darum gehen, möglichst genau die Form adresslistenbasierter Kommunikation zu beschreiben und $\mathrm{zu}$ verstehen. Hier geht es generell um die Konstituierung von Interaktionssystemen (abwesender) Anwesender in der Gesellschaft. Dabei wird vor allem die eigentümliche Bewältigung doppelter Kontingenz durch Adresslistenkommunikation im Fokus stehen. Das im Vergleich mit Kommunikation Anwesender (Face-to-Face Kommunikation) innovative Potential adresslistenbasierter Kommunikation zeigt sich nämlich, wie detailliert $\mathrm{zu}$ erörtern sein wird, gerade im unterschiedlichen Umgang mit dem Problem doppelter Kontingenz. Entsprechend orientiert sich vorliegende Studie methodisch an der funktionalen Analyse (vgl. Luhmann, 1984, S. 83 ff., 1997, S. $36 \mathrm{ff}$.). Durch funktionalen Vergleich mit Kommunikation unter Anwesenden (in Bezugnahme auf eine kanonische Studie von Kieserling, 1999), wird möglich, charakteristische Funktionen und (latente) Strukturen von internetbasierter Adresslistenkommunikation - sozusagen Kommunikation unter abwesenden Anwesen$d e n^{3}$ - herauszuarbeiten.

Im Zuge der Ausführungen des ersten Teils, wird zu erläutern sein (Abschnitt I. 5), dass sich mit der Kommunikation in Adresslisten in der «digitalen Moderne» ein neues Kommunikationsmedium ausdifferenziert hat. Nämlich technisch generalisierte Kommunikationsmedien. Dies in Anlehnung an die bereits von Luhmann erläuterten symbolisch generalisierten Kommunikationsmedien (vgl. Luhmann, 1997, S. 316 ff.; spez. etwa Luhmann, 1992, in Bezug auf Wahrheit und 1994 in Bezug auf Geld).

Im zweiten Teil (II.) werden, am Fallbeispiel der Anbahnung von (romantischer) Liebe durch Adresslisten, die eher abstrakten Überlegungen des ersten Teils angewendet und veranschaulicht. Im Rahmen vorliegender Untersuchung ist das symbolisch generalisierte Kommunikationsmedium Liebe von besonderem Interesse, weil bei adresslistenbasier-

3 Die Rede von "abwesenden Anwesenden» trägt dem Umstand Rechnung, dass Kommunikation von räumlich Abwesenden in Adresslisten nicht notwendig zeitlich synchronisiert erfolgt, gleichwohl diese Möglichkeit durch "Instant-Messaging" (oft) besteht. Dies ist insbesondere auch bei privat erstellten Adresslisten der Fall, die durch Software Applikationen ("Apps", wie etwa WhatsApp, Line, Skype) ermöglicht werden. 
ter Kommunikation gerade in Bezug auf höchstpersönliche Kommunikation äusserst optimistische Erfolgserwartungen vorherrschen. ${ }^{4}$ Insofern geht es im zweiten Abschnitt darum, zu zeigen, wie die für Liebeskommunikation charakteristischen Strukturen (wie von Luhmann 1982 dargelegt) durch Medien der Adresslisten aufgegriffen, bzw. berücksichtigt werden. Es kann vermutet werden, dass sich der Erfolg, bzw. die Popularität von Singlebörsen wesentlich aus dieser gelingenden Bezugnahme erklärt.

Im abschliessenden dritten Teil (III.) wird erläutert, wie durch Adresslistenkommunikation Reputation in ihrer Multifunktionalität zunehmend an gesellschaftlicher Bedeutung gewinnt.

\section{Form adresslistenbasierter Kommunikation}

\section{Adresslisten als Medien}

Adresslisten werden, wie schon angedeutet, vorliegend als Adress-Medien verstanden. Also als eine Vielzahl von lose gekoppelten, gleichartigen Elementen, als die die Nutzerprofile in Adresslisten gelten sollen, welche potentiell in Form gebracht, also strikt gekoppelt werden können (grundlegend: Heider, 1926; in systemtheoretischer Ausarbeitung Luhmann, 1997, S. $190 \mathrm{ff}$.). Als Formung des Mediums, als «strikte Koppelung» soll erwartungsgenerierende Kommunikation zwischen den Adressaten bzw. Adressanten ${ }^{5}$ von Adresslisten gelten.

4 Es ist hier von erstaunlich hohen Nutzerzahlen auszugehen. So lag Ende 2015 die Anzahl der Mitgliedschaften bei Online Dating Börsen in Deutschland bei 118.1 Millionen; die Zahl der aktiven Nutzer lag immerhin noch bei 8.4 Millionen (Quelle: Statista: Statistiken zum Online Dating, https://de.statista.com/ themen/885/online-dating/).

5 Wir nutzen hier den veralteten Begriff «Adressant» (statt Absender oder Sender) zur Abgrenzung vom Sender-Empfänger Model der Kommunikation. Im systemtheoretischen Sinne ist Kommunikation als selbstreferentielle Operation sozialer Systeme zu verstehen («Kommunikation kommuniziert»), als eigendynamische Operation autopoietischer Systeme. Kommunikation wird also nicht als
Dies mag anfänglich eine lediglich durch einen "Button" realisierte «Freundschaftsanfrage» (Facebook) sein; später etwa Chats, Sprachnachrichten, (Bild-)Telefonate, Face-to-Face-Kommunikation.

Von Adresslisten als Medien kann sicher bei öffentlichen Adresslisten (wie Parship, eDarling, Tinder, LinkedIn, Joinmytrip usw.), deren Zugang durch Mitgliedschaft allerdings oft erkauft werden muss, die Rede sein. Dies im Gegensatz zu privaten Adresslisten, die sich in Mobiltelefonen und durch Messenger-Apps, wie WhatsApp, Facebook messenger, Line usw. ${ }^{6}$, erstellen lassen. Die Elemente (Adressen) privat erstellter Adresslisten sind üblicherweise durch unterschiedliche Erwartungen strukturiert, vorgeformt, wodurch sich schon vorweg ausdifferenzierte Kommunikationspräferenzen ergeben. Flüchtige Bekanntschaften von Personen, deren Namen kaum noch erinnert wird, sind neben Arbeitskollegen, Freunden, die auch regelmässig Face-to-Face getroffen werden, Geschäftspartnern, Sportfreunden, (Ex-)Lebenspartnern, dem Ehepartner, Liebschaften, Reisebekanntschaften usw., aufgelistet. Bei privat erstellten Adresslisten kann also nicht ohne weiteres von der Gleichartigkeit der Elemente, einem medialen Charakter ausgegangen werden. ${ }^{7}$

Austausch von Nachrichten, bzw. Informationen zwischen Sendern und Empfänger verstanden (vgl. Luhmann, 1984, S. $191 \mathrm{ff}$.).

6 Für einen Überblick an Anwendungen: siehe den Wikipedia Artikel «Liste von mobilen Instant-Messengern».

7 Allerdings ist auch nicht auszuschliessen, dass private Adresslisten im Laufe jahrelanger Nutzung den Charakter eines Mediums bekommen können, also in der dann oft undifferenzierten schlichten Vielzahl von Einträgen den Charakter von «öffentlichen» Adresslisten annehmen. Nutzer von Facebook etwa verfügen über durchschnittlich 342 «Freunde» (gemäss statistischen Untersuchungen - vgl. Data Science of the Facebook World, 14.4.2013 - http://blog. stephenwolfram.com/2013/04/data-science-of-the-facebook-world/) Festzuhalten ist deshalb, dass zumindest fallweise nachfolgende Überlegungen auch für privat erstellte Adresslisten Geltung beanspruchen können. 


\section{Das Problem der doppelten Kontingenz}

Im Problem der doppelten Kontingenz, welches das Verhältnis von Ego und Alter betrifft, stellt sich das Grundproblem der Ermöglichung sozialer Handlungen - ja sozialer Ordnung. In den vielfältigen Lösungsmöglichkeiten dieses Problems realisiert sich soziales Handeln. Wobei «Lösung» hier nicht als Beseitigung des Problems doppelter Kontingenz zu verstehen ist (was Systeme kollabieren lassen würde), sondern davon ausgegangen wird, «dass doppelte Kontingenz zwangsläufig zur Bildung von sozialen Systemen führt und in diesem Sinne als Dauerproblem (nicht nur: als Anstoss) autokatalytisch wirkt (...).» (Luhmann, 1984, S. 177)

Das Problem stellt sich erst dadurch, dass sich Alter und Ego gegenseitig und gleichzeitig als Sinnsysteme erleben; dies durch die Intransparenz selbstreferentiell geschlossener Bewusstseine, die sich gewissermassen als "black boxes" (vgl. Luhmann, 1984, S. 156) erfahren. Denn «[z]u einem Akutwerden doppelter Kontingenz genügt (...) nicht die blosse Faktizität der Begegnung; zu einem motivierenden Problem der doppelten Kontingenz (und damit: zur Konstitution sozialer Systeme) kommt es nur, wenn diese Systeme in spezifischer Weise erlebt und behandelt werden: nämlich als unendlich offene, in ihrem Grunde dem fremden Zugriff entzogene Möglichkeiten der Sinnbestimmung» (Luhmann, 1984, S. 151-152).

Indem Alter und Ego (als alter Ego) im gegenseitigen Erleben ihre Erwartungen an die Erwartungen des je anderen knüpfen (und so als doppelt kontingent erfahren), wird - idealtypisch - in unbestimmbarer Selbstreferenz jegliches Handeln blockiert. Anfängliches Handeln, bar jeglicher Orientierung, ist derart mit maximaler Erwartungsunsicherheit verbunden, also höchst risikoreich. Es entsteht ein Zirkel, der erst aufzulösen, gewissermassen $\mathrm{zu}$ enttautologisieren ist, damit überhaupt etwas passiert: «Ich tue, was Du willst, wenn Du tust, was ich will» (Luhmann, 1984, S. 166). In der Konstituierung sozialer Systeme aus dieser Unbestimmtheit, in der Überwindung des Problems der doppelten Kontingenz, etwa durch Etablierung von Interaktionssystemen, wird anfängliche maximale Erwartungsunsicherheit in zunehmende Erwartungssicherheit verwandelt (vgl. auch Kron et al. 2003, S. 376-377). Zunächst risikoreiche, so allerdings Aufmerksamkeit fördernde Unbestimmtheit der Begegnung doppeltkontingenter Systeme ermöglicht, dass anfänglich jeder Zufall, ein flüchtiger Blick, eine kaum wahrnehmbare Geste, ein Irrtum etwa, die Situation enttautologisieren kann (Luhmann, 1984, S. 165-166).

Dabei ist festzuhalten, dass Systembildung nur erfolgen kann, wenn Unterschiede nicht aufgelöst werden, sondern Unterscheidungen vielmehr operativ fortlaufend $\mathrm{zu}$ neuen Unterscheidungen führen. In Bezug auf soziale Systeme kann Kommunikation deshalb nicht gleichbedeutend sein mit der Übertragung von Information, gewissermassen einem "whitening" der "black boxes," als die Alter und Ego sich erfahren. Kommunikation würde dadurch zum Erliegen kommen. Es ist gerade die Unmöglichkeit der Kommunikation die Intransparenz von Ego und Alter durch Kommunikation aufzulösen, die Kommunikation "autokatalytisch" ständig re-vitalisiert. Die gegenseitige Intransparenz von Bewusstseinssystemen (Ego/Alter) und sozialen Systemen (etwa Interaktionssystemen) - also die Stabilität dieses Unterschieds als Unterschied -, sorgt für den Fortgang (die Autopoiesis) sowohl von gedanklichen, wie auch von kommunikativen Unterscheidungen. In sich gegenseitig konstituierender autopoietischer Selbstreferenz kann weder Kommunikation Kommunikation, noch können Gedanken Gedanken transzendieren. Systembildung ist in diesem Sinne als der beständige Versuch der Lösung eines unlösbaren Problems zu verstehen. ${ }^{8}$

8 Dem widerspricht nicht, dass in verständnisvoller («harmonischer») Kommunikation, dann wohl oft im Dunstkreis von Kommunikation im Medium der Liebe oder der innigen (sic) Freundschaft, gleichwohl der (bewusste) Eindruck von Transparenz erzeugt werden kann, der Eindruck eines gegenseitigen «vollkommenen» Verständnis'. Gerade weil dieser Zustand des Bewusstseins nicht 


\section{Bewältigung des Problems doppelter} Kontingenz durch Adresslisten

\subsection{Kontrolle von \\ Adresslistenkommunikation durch \\ Komplexitätsreduktion}

Bei Kommunikation im Medium der Adresslisten handelt es sich um eine besonders effiziente, technisch unterstützte Möglichkeit, den Zirkel doppelter Kontingenz aufzulösen. Kommunikation abwesender Anwesender in digitalen Adresslisten, eine unverbindliche Mitteilung zunächst, ist fast risikolos, ohne zu erwartende negative Folgen möglich. Etwa anfänglich lediglich als «technisch», mit Hilfe eines "Buttons" erfolgendes «Zuzwinkern» oder «Interesse». Demnach möglich, ohne allfällige Peinlichkeit, wie sie in Face-toFace-Kommunikation wahrscheinlich wäre, würden hier die vergleichsweise rüden Methoden der Kontaktaufnahme besonders aber der Verweigerung der Aufnahme eines Kontakts - angewendet, wie sie das Medium der internetbasierten Adresslisten erlaubt. Ein unmittelbares Verweigern, bzw. Ignorieren von Antworten, etwa auf «Freundschaftsanfragen» (Facebook), nicht unüblich in adresslistenbasierter Kommunikation, ist in der Kommunikation physisch Anwesender fast unmöglich, bzw. wird als barscher, feindseliger Fortgang von Kommunikation verstanden.

Charakteristisch für Kommunikation in Adresslisten ist eine massive Einschränkung von körperlicher Wahrnehmung. Hingegen ist für Kommunikation unter Anwesenden gerade die informationsgenerierende Reflexivität von körperlicher Wahrnehmung (Blickkontakte etwa ermöglichen die Beobachtung eigener

mitgeteilt werden kann (Sprache erlaubt, ja evoziert leichthin Negation; Mitteilungen würden diesen Zustand also destruieren), wird er allenfalls etwa durch vielsagendes (nichtssagendes?) versonnenes Schweigen, tiefe Blicke, einen verständnisvollen Händedruck kommuniziert. Mehr bedarf es eben aufgrund tiefen Verständnis' nicht. Und wir, als Beobachter der sich so Beobachtenden, können zudem beobachten: Mehr darf es auch gar nicht bedürfen.
Beobachtung) kennzeichnend (vgl. Kieserling, 1999, S. $110 \mathrm{ff}$.). Das reichhaltige, informierende Anschauungsmaterial, das die Wahrnehmung der Physis liefert, entfällt. Lediglich das Aussehen in Form von Bildern (immerhin eingeschränkte körperliche Wahrnehmung), ein gemeinsames Reiseziel, gemeinsame Bekannte, ähnliche berufliche Interessen, mögen zunächst als Attraktoren für eine Kontaktaufnahme dienen. Informationen, wie die Wahl der Kleidung, Stimme, Wortwahl und Rhetorik, das Verhalten in der Kommunikation, direkter oder indirekter Blickkontakt, Wendigkeit/Schlagfertigkeit in der Kommunikation, Erröten, durch Auftreten indizierte Selbst(un)sicherheit usw., bleiben notwendig aussen vor. Die Komplexität von schriftlicher (und in schwächerem Masse auch fernmündlicher) Kommunikation ist hingegen in ihrer Form extrem reduziert; sie erfolgt in notwendig serieller Selektivität, in der der Verkettung von Information, Mitteilung und Verstehen. ${ }^{9}$

Es ist die massive Einschränkung der körperlichen Wahrnehmung, die die Enttautologisierung des Zirkels doppelter Kontingenz erleichtert. ${ }^{10}$ Der sich über physische Wahrnehmung ergebende dichte (Zusatz-)Gehalt an Information (etwa sich in Stottern und Erröten ausdrückende Unsicherheit, Übergewicht, Ticks, die keiner bewussten Kontrolle unterstehen usw.) ist in der Kommunikation körperlich Anwesender kaum zu kontrollieren. ${ }^{11}$ Die

9 Wir argumentieren hier genau entgegengesetzt zu Illouz (2011, S. $409 \mathrm{ff}$.), die von einem dichten Informationsgehalt schriftlich basierter Internet-Kommunikation ausgeht, von einem ausgedünnten, «lllusionen» erlaubenden, bei Einbezug von körperlicher Wahrnehmung.

10 Und dies paradoxer Weise gerade in Bezug auf die Anbahnung von Liebeskommunikation, wo es doch um die Universalbetreuung eines Individuums geht. Aber: Liebe auf den ersten Blick ist - der erwähnenswerte - Ausnahmefall. Indifferenz, und allenfalls sogar Ablehnung, der Normalfall. Vgl. zur Kommunikation im Medium der Liebe Abschnitt II.

11 Auch die Versendung von "Emoticons," die das sich körperlich, etwa über das Gesicht ausdrückende Gefühlsleben von Adressat bzw. Adressant kennzeichnen sollen, bie- 
massive Einschränkung der informativen Möglichkeiten erleichtert abwesenden Anwesenden die Kontrolle, nicht zuletzt auch darüber, ob Kommunikation überhaupt initiiert wird. Die zunächst gegebene Einschränkung körperlicher Wahrnehmung bietet nämlich die Möglichkeit, diese kontrolliert zu steigern bzw. einschränken. Und damit den Grad der Erwartungs(un) sicherheit der Kommunizierenden sowohl auszudrücken, wie auch zu bekräftigen bzw. abzuschwächen. Die Intensivierung der Koppelung ${ }^{12}$, ein erhöhtes Mass an Vertrauen bzw. Erwartungssicherheit, welche sich auch im zunehmenden Mass ermöglichter körperlicher Wahrnehmung sowohl ausdrücken, wie auch verstärken kann, erfolgt gewissermassen performativ selbstgesteuert, durch den je erreichten Grad der Koppelung selbst: «(...) Vertrauen [hat nämlich] jenen zirkulären, sich selbst voraussetzenden und bestätigenden Charakter, der allen Strukturen eigen ist, die aus doppelter Kontingenz entstehen. Es macht Systembildungen möglich und gewinnt aus ihnen dann wieder die Kraft zu verstärkender, riskanterer Reproduktion» (Luhmann, 1984, S. 181, Hervorhebung durch den Autor). ${ }^{13}$

ten hier natürlich keine Abhilfe. "Emoticons" sind als Elemente der Kontrolle von Kommunikation zu verstehen und nicht als Hilfsmittel, um unverstellten Gefühlen der kommunizierenden Personen Ausdruck zu verleihen.

12 Als Minimalform strikter Koppelung soll hier gelten, dass auf eine Mitteilung zumindest eine Anschlussmitteilung des zunächst Adressierten zurück an den Adressanten erfolgt, also davon ausgegangen werden kann, dass erwartungsbildend zumindest einmalig Information von Mitteilung unterschieden und verstanden worden ist. Im Zusammenhang mit Medien der Adresslisten können dies auch "Likes"|“Dislikes," "ReTweets," Kennzeichnung als «Favoriten» usw. sein.

13 Der Zuwachs an Kontrolle ist anfällig dafür, überschätzt zu werden. So wird bei allmählich intensivierten, Vertrauen scheinbar zu kontrollierenden Kontaktaufnahmen eben oft unterschätzt, dass Identität im Internet durch eingeschränkte, bzw. leicht zu kontrollierende körperliche Wahrnehmung gefälscht werden kann. Dies erleichtert diverse Formen der Kriminalität, etwa Betrug finan-
In kontrollierter Steigerung (oder allenfalls auch Verweigerung) körperlicher Wahrnehmung kann es, etwa in Datingwebseiten, von Chatkommunikation zur Versendung von E-Mails kommen; von E-Mails zum Austausch von Bildern; von Bildern zu Telefonaten; von Telefonaten zu Bildtelefonaten (etwa über Dienste wie «Skype» oder «WhatsApp»); von Bildtelefonaten zu Treffen Face-to-Face; von Treffen Face-to-Face zu körperlichen Intimitäten. Wobei natürlich dem Einzelfall überlassen bleibt, Geschwindigkeit und Schrittfolge der so graduierten Koppelung, bzw. allenfalls auch kommunikativen Rückzug oder gar Abbruch, zu bestimmen.

Nicht nur die Reduktion von Komplexität durch eingeschränkte körperliche Wahrnehmung erlaubt eine, jedenfalls im Vergleich zur Kommunikation physisch Anwesender, überragende Kontrolle von Kommunikation, sondern auch der für Adresslistenkommunikation typische Sachverhalt, dass raum-zeitliche Parameter zur Disposition stehen. Typisch für schriftliche, und damit auch für Adresslistenkommunikation ist, dass die Unterscheidung (das Verstehen) der Unterscheidung von Information und Mitteilung zeitlich und räumlich auseinandergezogen ist. Speichermedien (wie Papyrus, Bücher, Computerserver) erlauben, dass Mitteilungen auch noch später und woanders von Informationen unterschieden, und so verstanden werden können. Zeit ist in Adresslistenkommunikation also nutzbar, etwa um besonders originelle, überlegte Anschlussmitteilungen auszuarbeiten, oder etwa um Zeit selbst latent informativ zu nutzen, etwa indem entschieden werden kann, ob umgehend oder zeitlich verzögert geantwortet wird. ${ }^{14}$

zieller Art, Pädophilie, Heiratsschwindelei, Stalking usw.

14 In der Diktion Bourdieus (1982) lässt sich feststellen, dass die Disposition der Adresslistenkommunikation ein effektives Management der Arten des «Kapitals», über das Individuen verfügen, erlaubt. So lässt sich etwa - gerade durch zunächst schriftliche Kommunikation - ein hohes kulturelles Kapital herausstellen, bei etwa gleichzeitigen Defiziten hinsichtlich des ökonomischen 
Hingegen ist zeitliche Kontrolle in Face-to-Face Kommunikation äusserst eingeschränkt. Anschlusskommunikation hat, soll sie nicht irritieren, innert angemessener Zeit zu erfolgen. Zu starke zeitliche Kontrolle (Monolog) ist, wenn es sich nicht um Vorträge handelt, denen zeitliche Freiheiten auch nur unter der Voraussetzung eines zeitlichen Rahmens zugestanden werden, bei der Kommunikation Anwesender eher unerwünscht. Zwar ist es in jedem Fall die Kommunikation selbst, die zeitlichen Erwartungsdruck aufbaut («Kommunikation kommuniziert»), im Vergleich zu Interaktionssystemen Anwesender ist dieser Druck in Adresslistenkommunikation jedoch weitaus weniger restriktiv, was ein stärker differenziertes, Kontrollmöglichkeiten erlaubendes Zeitmanagement erlaubt.

Nicht zuletzt ist das Risiko von (anfänglicher) Kommunikation im Medium der Adresslisten auch durch die schlichte Vielzahl potentieller Kontakte minimiert. Werden Mitteilungen ignoriert, stehen weiterhin hunderte andere Elemente $\mathrm{zu}$ Verfügung, um Formen der strikten Koppelung zu realisieren. ${ }^{15}$

\subsection{Funktionale Äquivalente zur Steigerung von Komplexität in Adresslistenkommunikation}

Die erhebliche Kontrolle von Adresslistenkommunikation ermöglichende Reduktion von Komplexität - durch eingeschränkte körperliche Wahrnehmung, weitgehende Beherrschung raum-zeitli-

Kapitals. Oder es wird, umgekehrt, etwa ein hohes ökonomisches Kapital betont (vgl. http://www.mysugardaddy.eu/), das ermöglicht, allenfalls später aufgedeckte Defizite hinsichtlich des kulturellen oder sozialen Kapitals zu überstrahlen.

15 Was allenfalls selbstverstärkende Effekte nach sich zieht. Wird ohnehin mit dem Ignorieren von Mitteilungen gerechnet, mag es generell sinnvoll erscheinen, zahllose unverbindliche (Massen-)Mitteilungen («Hallo», «Wie geht's?», «Du hast ein nettes Lächeln ...» usw.) zu versenden, was zur Folge hat, dass generell mit unverbindlichen Mitteilungen gerechnet werden muss, die mit hoher Wahrscheinlichkeit ignoriert werden können ... chen Faktoren und durch die Vielzahl alternativadressabler Adresslistenmitglieder (Nutzerprofile) - bedarf funktionaler Äquivalente, um kommunikative Komplexität wiederum zu steigern. Denn es ist davon auszugehen, dass etwa die auf Dauer eingeschränkte körperliche Wahrnehmung abwesender Anwesender, etwa als Chatoder E-Mail-Partnerschaften, destabilisierend wirkt. Die Möglichkeit, dass sich durch dominantere körperliche Wahrnehmung (bei Preisgabe auch zeitlicher Kontrollmöglichkeiten) der Informationsgehalt der Adresslistenkommunikation steigern (bzw. allenfalls auch einschränken) lässt von Chats zum Austausch von Bildern, von Telefonie zur Videotelefonie usw. -, wirkt in diesem Sinne stabilisierend.

Ein weiteres funktionales Äquivalent, adresslistenbasierte Kommunikation wieder mit Komplexität anzureichern, ist, dass die Historie der gesendeten Nachrichten gespeichert wird, bzw. gespeichert werden kann. Damit spielt die «Systemgeschichte» bei Kommunikation im Medium der Adresslisten gleich zu Beginn eine entscheidende Rolle; dies zumal die Historie der Mitteilungen, technisch unterstützt, exakt abgespeichert wird. Bei Kommunikation unter Anwesenden ist davon auszugehen, dass die Systemgeschichte erst im Laufe der Zeit eine zunehmend grössere Rolle spielt, also «(...) die Interaktion im Laufe der Zeit Prämissen aufbaut, die erschwert negierbar sind, (...) in Form einer Systemgeschichte, die allen Beteiligten unverstellbar präsent ist, so dass niemand die eigenen Beiträge dazu einfach bestreiten oder sich aus den Erwartungen, die dadurch aufgebaut wurden, einfach zurückziehen kann, ohne dem Widerstand der anderen zu begegnen. Diese Prämisse ist dann zwar im System selbst konstituiert, gleichwohl aber Vorgabe für jede Einzeloperation und durch weitere Kommunikation nicht mehr in vollem Umfange auflösbar» (Kieserling, 1999, S. 134).

Durch die Einschränkung (auch reflexiv wirksamer) körperlicher Wahrnehmung ist zudem die Möglichkeit von indirekter Kommunikation - also etwas «fallenzulassen», «verstehen zu geben», etwas «durchblicken zu lassen» (vgl. Kie- 
serling, 1999, S. 147 ff.) -, welche die Kommunikation unter Anwesenden auszeichnet, extrem eingeengt. Auch wenn sich das Problem der Initiierung von Kommunikation, für die indirekte Kommunikation bei der Konstituierung von Interaktionssystemen unter Anwesenden nutzbar gemacht werden kann, ${ }^{16}$ in adresslistenbasierter Kommunikation kaum stellt, können die gespeicherte Systemgeschichte der Nachrichten und die Möglichkeit, dass die strikte Koppelung der Personen von Adresslisten potentiell steigerbar ist, als funktionelle Äquivalente für komplexitätsinduzierende körperliche Wahrnehmung verstanden werden. ${ }^{17}$

So ermöglicht einerseits die Steigerung der Erwartungssicherheit der Kommunikation von Nutzern von Adresslistenmedien auch «strikte Koppelungen» bis hin zur Face-to-Face Kommunikation, und damit potentiell auch indirekte Kommunikation unter Anwesenden. Die Speicherung der Systemgeschichte (von Chats oder E-Mails) ermöglicht andererseits, dass sich im Zuge von Rekursen auf die Systemgeschichte - in der Systemgeschichte und wiederum als Systemgeschichte - Sinngehalte (Bedeutungen) der Systemgeschichte immer wieder ändern; also bereits Ge-

16 So wird «(...) indirekte Kommunikation verwendet (...), um das Problem der Initiative $\mathrm{zu}$ entschärfen. Die Problemlösung läuft dann über eine Ambiguisierung der Zurechnungsfrage. Vor allem bei ungleich verteilten Initiativrechten gilt dann: Wer Initiative zeigen müsste, den kann man durch indirekte Kommunikation dazu ermuntern. Reflexives Wahrnehmen macht es leicht so $\mathrm{zu}$ verfahren. Blicke können länger dauern als üblich. Ein Lächeln kann mehr als nur Standardfreundlichkeit ausdrücken. Man kann einen Fehler und die Entschuldigung für den Fehler (zu der man eben dadurch ein Recht erwirbt) zur Andeutung eigener Gesprächsbereitschaft nutzen» (Kieserling, 1999, S. 162-163) usw.

17 In privaten Adresslisten kann auch die Ambiguität der Adressierung von Kommunikation in Gruppenchats als Form der indirekten Kommunikation verstanden werden. So kann etwa Kommunikation scheinbar an die Gruppe gerichtet sein, gleichwohl so einem einzelnen Gruppenmitglied etwas «durch die Blume» mitgeteilt werden soll. sagtes in neuem Licht erscheint, sich neu Erwähntes relativiert, Widersprüche auftauchen oder abgeschwächt werden usw. ${ }^{18}$ Gleichwohl die sich ergebenden Mehrdeutigkeiten, die Unsicherheiten von Bedeutungen, im Rückgriff auf die schriftlich festgehaltene Systemgeschichte objektiver, also, im Vergleich zur Kommunikation unter Anwesenden, allenfalls schwerer $\mathrm{zu}$ kontrollieren sind, können diese doch als funktionales Äquivalent zu komplexitätssteigernder indirekter Kommunikation gelten.

Im Vordergrund steht dabei allerdings nicht die Funktion der Initiierung von Kommunikation; dieses Problem ist in adresslistenbasierter Kommunikation bereits auf effiziente (technisch unterstützte) Weise gelöst. Gerade die Leichtigkeit aber mit der Interaktionssysteme abwesender Anwesender durch Adresslisten initiiert werden können, hat anfänglich zur Folge, dass sich Kontakte derart durch Flüchtigkeit, Beliebigkeit, Unverbindlichkeit - ja: Stereotypizität, auszeichnen. Die Möglichkeit der Steigerung der strikten Koppelung und die Speicherung der Systemgeschichte, haben die Funktion, die Komplexität dieser Interaktionssysteme $\mathrm{zu}$ steigern, um eine höhere Verbindlichkeit bzw. Stabilität zu ermöglichen. Dabei ist es im Laufe der Systemgeschichte nicht unbedingt eine sukzessive Erhöhung des Vertrauens bzw. der Sicherheit von Erwartungen, die für eine Stabilisierung von Interaktionssystemen sorgt. Im Gegenteil, sich im Rekurs auf die Systemgeschichte ergebendes Misstrauen und ergebende Erwartungsunsicherheit, angesichts von Widersprüchen, Unstimmigkeiten, Ambivalenzen, Unverständlichem, kann zu mehr Engagement, höherer Aufmerksamkeit, mehr Vor- oder Nachsicht, stärkerem Einsatz, schlicht zu intensiverer Kommunikation in Entgegnung von Irritationen sorgen und damit

18 Wenn Sinn als Medium, als Einheit der Differenz von Aktualität und Potentialität verstanden wird (Luhmann 1984, S. $92 \mathrm{ff}$.), verändern sich Formen des Sinns (Bedeutungen), Unterschiede ausmachende Unterschiede zwischen aktuellen und potentiellen Möglichkeiten, im Zuge der Systemgeschichte, also mit sich ändernder Aktualität. 
die Stabilität derartiger Interaktionssysteme steigern.

Interaktionssysteme, ob unter Anwesenden oder unter «abwesenden Anwesenden» (z.B. Chatpartner), zeichnen sich als autopoietische Systeme durch grundlegende, die Ebene der Operationen, also Kommunikation betreffende Selbstreferentialität aus («Kommunikation kommuniziert»). Es bedarf demnach der Enttautologisierung, der Selbstreferenzunterbrechung oder Asymmetrisierung, um diese Systeme zu erhalten (vgl. Kieserling, 1999, S. 187 ff.) Dabei bedingt die Abwesenheit der Wahrnehmung der Physis, dass die Selbstreferenzunterbrechung in adresslistenbasierter Kommunikation im Wesentlichen zunächst thematisch erfolgt, also hinsichtlich der sachlichen Sinndimension über Externalisierung. Erst später ist möglich, dass Enttautologisierung auch über die Sinndimension der Zeit, also im Rekurs auf die Systemgeschichte eines Interaktionssystems, und die soziale Sinndimension, etwa in der Ausdifferenzierung geschlechterbezogener Rollenbilder, durch Expertentum, Dominanz usw., erfolgt. Nicht zuletzt ist davon auszugehen, dass die Selbstreferenzunterbrechung abwesender Anwesender (also chatbasierte Interaktionssysteme oder etwa "E-Mail-Buddies" ${ }^{19}$ ) zudem über Reflexivität erfolgt. Es ist charakteristischer Weise der durch Speicherung mögliche Zugriff auf die exakte Historie dieser Interaktionssysteme, der Selbstreferenzunterbrechung durch Reflexivität privilegiert.

Dass eine Asymmetrisierung bei adresslistenbasierter Kommunikation thematisch erfolgt, ergibt sich schon aus der Tatsache, dass die Adresslisten selbst thematisch organisiert sind; etwa als Datingpartnerschaften jedweder Couleur (Liebe,

19 Da zumindest synchrone Anwesenheit Bedingung von Interaktionssystemen ist, kann E-Mail-Interaktionssystemen dieser Status nur dann zugerechnet werden, wenn klar ist (etwa durch umgehende Beantwortung von Mitteilungen, Statusanzeigen), dass der andere synchron anwesend ist. So im Unterschied etwa zu Brief-Freundschaften.
Freundschaft, Sexualität ${ }^{20}$, Seitensprung usw.), als potentielle Reisebekanntschaften, berufliche Netzwerke, Freundschaften mit thematischen Schwerpunkt (Autos, Motorräder, Sport usw.), Lernpartnerschaften, usw. Thema und Interesse an demselben, indiziert durch die Registrierung in der betreffenden Adressliste, sind schon, bevor überhaupt Kommunikation erfolgt, klar festgelegt, was die Handhabung des Zirkels doppelter Kontingenz ungemein erleichtert. Dies zumal, angesichts der Abwesenheit von physischer Wahrnehmung, zunächst Kommunikationsofferten, bzw. darauf folgende Kommunikation, in weitgehender Anonymität erfolgen kann. So kann davon ausgegangen werden, dass Interaktionssysteme, die unter der Ägide einer Thematik stehen, leichter auszudifferenzieren sind, als bei bereits konstituierten Interaktionssystemen spezifische «Thematisierungsschwellen» (Luhmann, 1981, S. 55 ff.) zu überwin-

20 Auf technisch besonders effiziente Weise bedient sich die Anwendung «Tinder» (http:// www.gotinder.com/) adresslistenbasierter Kommunikation. Das Programm ermöglicht es Nutzern, anhand ihrer Profilbilder eine Vorauswahl der Personen zu treffen, die sie (erotisch) attraktiv finden und kennen lernen möchten. Ablehnung bzw. Zustimmung wird in der Smartphone-App durch ein «wegwischen» der Bilder nach links bzw. rechts, bzw. das Drücken entsprechender "Buttons" signalisiert. Die (je individuelle) Liste der Personen, die sich gegenseitiges Interesse gezeigt haben, wird daraufhin den Nutzern zu Verfügung gestellt, gefiltert nach der Entfernung der Personen unter Ausnutzung des GPS-Systems des Smartphones, bzw. der angegebenen Verortung der Nutzer. Kommunikation wird so wahrscheinlich gemacht, da eine symmetrische Asymmetrisierung in sachlicher (erotische Partnerschaft) und sozialer Hinsicht (gegenseitiges Interesse) bereits erfolgt ist, obwohl eine Kommunikationsofferte allenfalls gegenüber dem Abstraktum "Adressliste», nicht jedoch an die Adresse einer individuellen Person erfolgte. Zudem bleiben den Nutzern die enttäuschenden Listen desinteressierter bzw. einseitig interessierter Personen unbekannt, bzw. lediglich latent bekannt. 
den. Was wiederum der Etablierung neuer Interaktionssysteme zugutekommt. ${ }^{21}$

Eine Enttautologisierung der Interaktionssysteme durch die Sinndimensionen der Zeit (Systemgeschichte), der Sache nach (Thema) und über Reflexivität (im Zugriff auf die Systemgeschichte), kann für eine beträchtliche Stabilität von adresslistenbasierten Interaktionssystemen, wie Chat- oder E-Mail-Partnerschaften, sorgen. Dass die Abwesenheit der körperlichen Wahrnehmung, bzw. die dadurch eingeschränkte (bzw. idealisierte) Sozialdimension gerade in der Konstituierung von Intimbeziehungen oft eher konstruktiv, als destruktiv erlebt wird, wie die Popularität von Singlebörsen zeigt, ist angesichts der sich ergebenden Kontrollmöglichkeiten verständlich.

Gerade angesichts stabiler, idealisierter Komplexe von Erwartungen, die unter abwesenden Anwesenden aufgebaut werden können, ist die Wahrscheinlichkeit allerdings hoch, dass bei der späteren prosaischen Wahrnehmung des Körpers (bzw. körperlichen Verhaltens) mit destruktiv wirkenden Enttäuschungen $\mathrm{zu}$ rechnen ist. Zudem sollen, modernen Erwartungen gemäss, nicht zuletzt als Errungenschaft des Feminismus, Geschlechterrollen in Interaktionssystemen im Medium der Liebe symmetrisch, als eine Beziehung unter Gleichen, ausdifferenziert werden (vgl. etwa Illouz, 2011, S. 318), was ein Zustandekommen von Liebes-Beziehungen angesichts von Hierarchien (etwa Professoren - Studentinnen), oder angesichts stark unterschiedlichen Alters, oder angesichts wirtschaftlichen oder sonstigen Abhängigkeiten, zumindest unwahrscheinlich machen soll. Dem modernen Wertekanon

21 Dies ermöglicht durch Adresslisten extrem unwahrscheinliche Formen von Interaktion. Man denke hier an so unwahrscheinliche Interaktionssysteme, wie sie durch Kannibalismus- oder Suizid-Foren ermöglicht werden. In diesem Sinne werden auch eher neue Interaktionssysteme, etwa zu einer «Domina» konstituiert, als entsprechende Thematisierungsschwellen (hier: sexuelle Präferenz) in bereits bestehenden Interaktionssystemen (angelehnt etwa an Partnerschaft oder Ehe) zu überwinden. nach soll in Bezug auf Intimbeziehungen von einer Asymmetrisierung doppelter Kontingenz durch die Sozialdimension abgesehen werden. ${ }^{22}$

\section{Die Bewältigung des Problems doppelter Kontingenz durch Adresslisten als Problem}

Das Paradigma der Konstituierung von Interaktionssystemen unter Anwesenden ist die Konfrontation von gegenseitig intransparenten Bewusstseinssystemen. In adresslistenbasierter Kommunikation, bei der Konstituierung von Interaktionssystemen unter abwesenden Anwesenden kommt hinzu, dass nicht nur das Bewusstsein als "black box" zu verstehen ist, sondern zudem die jeweilige Adressliste der Adressaten bzw. Adressanten. Auch wenn Adresslisten, anders als Bewusstsein keine autopoietischen Systeme, nicht $a k t i v$ «(...) dem fremden Zugriff entzogenen Möglichkeiten der Sinnbestimmung» (Luhmann, 1984, S. 152) vorhalten können, haben die Teilnehmer von Kommunikation in Adresslisten zu berücksichtigen, dass Kommunikationsofferten, bzw. -annahmen in Relation (etwa Konkurrenz oder Widerspruch gar) $\mathrm{zu}$ anderen Offerten, bzw. Annahmen von anderen Elementen (Personen) der Adresslisten stehen und somit passiv gleichfalls «dem fremden $\mathrm{Zu}$ griff entzogene Möglichkeiten der Sinn-

22 Angebote, die die Enttautologisierung von Interaktionssystemen spezifisch auf Asymmetrien in der Sozialdimension stützen (etwa hinsichtlich Alters und ökonomischer Situation: http://www.mysugardaddy.eu/), werden dadurch zumindest moralisch fragwürdig, aber vermutlich, angesichts modernem moralischen Pluralismus', kaum weniger erfolgreich. Selbstredend kann es sich hier anfänglich nicht um Liebe handeln, geht es doch in diesen Fällen nicht um Individuen, sondern ihnen zuzurechnende spezifische Eigenschaften. So etwa Unterschiede hinsichtlich des «Kapitals» (Bourdieu) von Personen. In Abschnitt II wird gezeigt, dass es die strukturellen Charakteristika von Liebeskommunikation selbst sind, die bedingen, dass die Enttautologisierung von höchstpersönlichen Interaktionssystemen (Liebesbeziehungen) nicht über die Sozialdimension erfolgen darf. 
bestimmung» bereithalten. Sich deshalb jederzeit etwa Prioritäten ändern können, kommunikative Annahmen oder Offerten unter Vorbehalt stehen, eigene Zusagen allenfalls revidiert werden müssen, mit Absagen gerechnet werden muss, Verbindlichkeiten in Frage stehen usw.

Im Fokus steht bei Kommunikation unter Anwesenden das Bewusstsein. Es sind kommunikative Handlungen, die sich hier als kontingent darstellen. Selbst wenn kommunikative Handlungen auf eine bestimmte (nicht unmögliche) Weise möglich, gleichwohl aber, nämlich gegenseitig bedingt durch die Bedingungen des je Anderen, nicht auf diese Weise notwendig sind, und Kommunikation als Handlung so blockiert wird, steht gemeinhin Adressant bzw. Adressat (Alter bzw. Ego) der Kommunikation nicht infrage. ${ }^{23}$ Bei Kommunikation unter Anwesenden ist im Allgemeinen klar, dass bestimmte Individuen kommunizieren.

Bei Kommunikation unter abwesenden Anwesenden stehen zudem die Adresslisten der Kommunizierenden im Fokus. Kontingent werden damit (und dies auf jeden Fall mit Blick auf öffentliche Adresslisten) die adressierten Individuen von Kommunikation. Es ist nicht unmöglich mit einer bestimmten Person in Kontakt zu treten, aber auch nicht notwendig angesichts der Vielzahl gleichartiger Elemente (Nutzerprofile) in Adresslisten. Dies zumal der zufällige Charakter von Kommunikation in Adresslisten offenkundig ist. Nicht unbedingt die Individualität einer Person steht im Fokus, sondern etwa die Tatsache, dass sie gerade online ist, oder das eine Person früher, als eine andere, auf eine Kommunikationsofferte antwortete, oder die räumliche Entfernung zu dieser Person günstiger ist, als zu jener, oder das für ein Treffen eine Terminabsprache mit der einen Person bequemer ist, als mit der anderen usw.

23 Eine gleichwohl bestehende Fragwürdigkeit kann - als indirekte Kommunikation - gerade dazu benutzt werden, das Problem doppelter Kontingenz zu enttautologisieren (vgl. Kieserling, 1999, S. 147 ff., auch weiter oben: Fussnote 16).
Die Leichtigkeit, mit der bei Kommunikation im Medium der Adresslisten das Problem der doppelten Kontingenz handhabbar ist, ist dadurch bedingt, dass nicht, wie bei Kommunikation unter Anwesenden, mit einzigartig wahrgenommenen Individuen kommuniziert wird, sondern mit Elementen eines Mediums. Es lässt sich sagen, dass hier anfänglich nicht Individuen, sondern Dividuen kommunizieren. Personen also, die als Elemente von Adresslisten mit vielen anderen Elementen strikt koppelbar sind und sich so gewissermassen als "teilbar» darstellen. ${ }^{24}$

Unter Anwesenden, also in Fällen, in denen sich Ego und Alter mit ihrem Bewusstsein gegenseitig als "black boxes" erleben, kann jede (kommunikative) Handlung, ein kurzer Blick etwa, ein verschämtes Lächeln, ein Gähnen, ostentatives Ignorieren, verschränkte Arme, ein bestimmter Kleidungsstil, usw., mit Bedeutung aufgeladen werden, ist als kommunikative Handlung individuellen Personen zurechenbar und damit, da eben auch Ablehnung individuell zuzuordnen ist, risikoreich. Hingegen sind anfängliche kommunikative Offerten in Adresslisten in ihrer Bedeutsamkeit, ihrem Risiko (was Konsequenzen betrifft) für Individuen extrem limitiert. Der Adressat der Kommunikation in öffentlichen Adresslisten ist zu Anfang gewissermassen das Medium, nicht ein Individuum. Gerade dieses anfänglich beschränkte Risiko ist es, das problemloses Initiieren von Kommunikation in Adresslisten ermöglicht.

24 Wir können bei dieser Begrifflichkeit an Fuchs (2007, S. IV. Die Listenförmigkeit des Menschen, Hervorhebung durch den Autor) anknüpfen. Fuchs konzeptuell allerdings eher im philosophischen (begriffsanalytischen) Zugriff, bezogen auf die Charakterisierung des Menschen in seiner eben fragwürdigen Individualität. Wir gehen hier konkreter von Dividualität im Sinne der Bestimmung von «Individuen» als Elementen in einem Medium (hier: Adresslisten) aus, beziehen uns - mit diesem Begriff - also ausdrücklich nicht auf die Charakterisierung der wie immer fragwürdigen Individualität des Menschen. Vgl. in diesem Zusammenhang auch Nietzsche (1954, S. 75). 
Zurückweisungen, ignorierte Kommunikationsofferten von Individuen sind schmerzlich, führen schon angesichts dieser Möglichkeit dazu, dass Offerten nur zögerlich erfolgen. Mit ablehnenden Kommunikationsannahmen oder ignorierten Kommunikationsofferten von Dividuen in Adresslisten ist jedoch plausibler Weise, angesichts der Leichtigkeit, mit der (selbst) Kommunikation initiiert werden kann, bzw. (selbst) kommunikative Offerten ignoriert, bzw. abgelehnt werden können, stets zu rechnen. Das Scheitern anfänglicher Kommunikation muss nicht persönlich genommen werden. Allerdings erzwingt wohl die systematische Ablehnung - oder zumeist eher noch die systematische Ignoranz-des Adresslistenmediums gegenüber kommunikativen Offerten dazu, dass der Adressant Defizite der eigenen Person zurechnet.

Individualität wird in Adresslisten erst im Zuge der Kommunikation, im Zuge der strikten Koppelung der Adresslistenelemente, der Dividuen konstruiert. Erst im Rekurs auf die dann tatsächlich je individuelle Systemgeschichte des sich konstituierenden Interaktionssystems, im spezifischen Zugriff des Interaktionssystems auf ein bestimmtes Thema, indem durch den Austausch von Bildern, durch (Bild-) Telefonate oder Face-to-Face-Kommunikation komplexitätssteigernd körperliche Wahrnehmung zugelassen wird, kann sich das Interaktionssystem stabilisieren. Adressat und Adressant, zunächst als Dividuen lediglich gleichartige Elemente in einem Medium, werden gegenseitig zu Individuen.

Es muss allerdings davon ausgegangen werden, dass in der Systemgeschichte von Interaktionssystemen, die sich durch adresslistenbasierte Kommunikation anbahnten, die (anfängliche) Kontingenz der Dividuen bzw. Individuen erinnert wird. Individualität wird dadurch ermöglicht, dass im Zuge der Stabilisierung eines Interaktionssystems das Paradox der anfänglichen Kontingenz der Kommunizierenden - von Dividualität zur Individualität - verdeckt wird. Die Kontingenz von Individualität - gewissermassen die Beliebigkeit von Einzigartigkeit -, die bei
Kommunikation im Medium der Adresslisten unverblümt vor Augen geführt wird, stellt sich bei adresslisteninitiierter Kommunikation als latent auftauchendes Paradox dar, welches so konstituierte Interaktionssysteme destabilisieren kann. Problematisch ist dies vor allem für Interaktionssysteme, die von der wechselseitigen Einzigartigkeit von Ego und Alter ausgehen müssen, also mit Blick auf (romantische) Liebesbeziehungen. ${ }^{25}$ Adresslisten führen vor Augen, dass es sowohl für Adressat wie Adressant Alternativen gibt. Mit (vermeintlicher) Leichtigkeit mit anderen Personen kommuniziert werden kann, andere Personen getroffen, andere Personen wichtig oder wichtiger, andere Personen «individueller» werden können. Begegnungen sind, führen Adresslisten brutal vor Augen, nicht von «schicksalhafter» Bedeutung, sondern zufällig und kontingent. $^{26}$

Dieses Problem ist hinsichtlich Adresslisten struktureller Art. Es ist durch das unlösbare Problem doppelter Kontingenz gegeben, das sich aus der wechselseitigen Intransparenz der Adresslisten von Alter und Ego ergibt. Dem Problem ist demnach nicht "psychologisch» zu entgegnen. Es reicht nicht aus, (sich selbst) mehr Willen $\mathrm{zu}$ zeigen, an sich selbst (therapeutisch) «zu arbeiten», zu versuchen, (Liebes-) Beziehungen etwa dadurch zu stabilisieren, indem die Individualität des Anderen mehr wertgeschätzt wird (in diesem Sinne zuzustimmen: Illouz 2011, S. $34 \mathrm{ff}$.). Die mittlerweile oft in Alltäglichkeit gegebene Adresslistenkommunikation nötigt Kommunizierenden auf, einzurechnen, dass sowohl mit Blick auf die eigene Adresse

25 Es entbehrt nicht der Tragik, dass, der Erfolg von Datingwebseiten indiziert dies, heutzutage sehr häufig versucht wird, Liebesbeziehungen gerade durch adresslistenbasierte Kommunikation zu initiieren. Also mit Adresslisten ein Medium in Anspruch genommen wird, das der Konstituierung von (romantischer) Liebe strukturell hinderlich sein kann.

26 Die Kontingenz und Instabilität moderner Liebesbeziehung führt eindrücklich Illouz (2011) vor. Wir werden im folgenden Abschnitt II. auf Kommunikation im Medium der Liebe zurückkommen. 
(als Adressant) wie auch mit Blick auf Adressierte, stets mit Alternativen $\mathrm{zu}$ rechnen ist. Andere Kommunikation, andere Personen (für Andere) bedeutsame(re) Möglichkeiten bereithalten können. Auch wenn wir in vorliegender Untersuchung vorrangig öffentliche Adresslisten im Blick haben, kann sich dieses Problem auch durch überbordende private Adresslisten in Smartphones stellen.

Das unlösbare Problem der doppelten Kontingenz muss also unlösbar bleiben. «Lösungen», wie Adresslistenkommunikation, führen dazu, dass das Problem in anderer Form wieder auftaucht. So könnten die erwähnten Probleme adresslistenbasierter Kommunikation dazu führen, dass auch hier Kommunikation blockiert wird. Eine Meta-Lösung, bzw. ein Meta-Problem für in Adresslisten auftauchende Kommunikationsblockaden aufgrund doppelter Kontingenz mag sein, hier Kommunikation nicht zu ernst zu nehmen, selbst nicht $\mathrm{zu}$ verbindlich $\mathrm{zu}$ sein, da hier mit der Unverbindlichkeit anderer zu rechnen, es demnach vernünftig ist, nicht zu verbindlich zu sein ... Erneut also: Wie diesen sich nunmehr ergebenden Zirkel der Unverbindlichkeit auflösen?27

Festzuhalten ist jedenfalls, dass adresslistenbasierte Kommunikation die Stabilisierung von Interaktionssystemen im Sinne von Bekanntschaft (unpersönlicher Kommunikation) privilegiert, etwa im Sinne der Initiierung und Aufrechterhaltung von Kontakten in beruflichen Netzwerken. Die Speicherung von Kontakten, die leichte Erweiterbarkeit von Adresslisten, die Möglichkeit, die Systemgeschichte schriftbasierter Interaktionen (Chats und E-Mails) exakt abzuspeichern, die (technisch unterstützt) problemlose Initiierung bzw. Beendigung von Kommunikation, ermöglicht dies.

\section{Die mediale Form von Adresslisten}

Es bleibt die Frage zu stellen, um welche Form des Mediums es sich bei Adresslisten

27 In Abschnitt III. wird erläutert, dass Reputation in Adresslistenkommunikation angestrebt wird, um der hier bestehenden Gefahr der Unverbindlichkeit von Kommunikation zu entgegnen. handelt. Als Medien fallen sie weder klar in die Kategorie der Verbreitungsmedien, die sich dem Problem der Erreichbarkeit von Kommunikation in der Gesellschaft widmen (vgl. Luhmann, 1997, S. $249 \mathrm{ff}$.), noch sind sie unzweideutig als symbolisch generalisierte Kommunikationsmedien, die sich dem Problem des Erfolgs, der erfolgreichen Erwartbarkeit von Kommunikation stellen (vgl. Luhmann, 1997, S. 316 ff.), $\mathrm{zu}$ verstehen. Nicht unplausibel ist, internetbasierte Adresslisten als technisch generalisierte Kommunikationsmedien $\mathrm{zu}$ verstehen. Es geht hier zwar darum, Kommunikation - erfolgreich - mit dann erwartbaren Folgen (Kontaktaufnahme bzw. Stabilisierung von Interaktionssystemen) $\mathrm{zu}$ versehen, geleistet wird dies jedoch nicht über symbolische, sondern technische Generalisierung.

Digitale soziale Medien sind abhängig von der Erzeugung einer möglichst grossen Anzahl von gleichartigen Elementen (Nutzerprofilen) auf der Basis von elektronischen Formularen. Die Gleichartigkeit der Elemente ermöglicht eine (statistische) Datenverarbeitung, die, in Verbindung mit einer technischen Infrastruktur (Internet), eine mühelose informative Koppelung, bzw. Entkoppelung der Nutzerprofile erlaubt. Die Funktionalität digitaler sozialer Medien ist also in essentieller Weise abhängig von den technischen Möglichkeiten, die das Internet und die elektronische Verarbeitung der Daten der Nutzerprofile bietet. Würden die Daten in analoger Form vorliegen (etwa als Listen in Papierform), und würde eine durch Personen erfolgende Vermittlung der Nutzerprofile (am Beispiel der klassischen Heiratsvermittlung) erfolgen, wäre es unmöglich, die Funktionalität der heutigen digitalen sozialen Medien aufrecht zu erhalten - es wäre schlicht unangemessen, bei Listen derart überhaupt von einem Medium der Kommunikation auszugehen. Von daher ist gerechtfertigt, hier von technisch generalisierten Kommunikationsmedien zu sprechen.

Die Generalisierung erfolgt dabei nicht über Symbole - symbolische Generalisierung kann allenfalls vorausgesetzt wer- 
$\operatorname{den}^{28}-$, sondern erfolgt technisch, durch die Nutzung von gleichen, bzw. gleichartigen Programmen, bzw. "Apps." Es ist von erstaunlich hohen Nutzerzahlen auszugehen. So verwenden (Stand: Januar 2016) monatlich etwa 1.65 Milliarden Personen Facebook, Messenger, wie WhatsApp oder Facebook messenger, werden von etwa je einer Milliarde Personen angewendet, die Fotosharing Plattform Instagramm verfügt über etwa 400 Millionen Nutzer. ${ }^{29}$ Dabei sind es die - eben programmatisch - beschränkten Möglichkeiten der Apps, die bestimmte Folgekommunikation wahrscheinlicher, bzw. erwartbarer machen, als andere.

Die generell durch die Operation der sprachlichen Negation gegebene Unwahrscheinlichkeit von Kommunikation wird also technisch (programmatisch) mit bestimmten erwartbaren Folgen versehen. Die Selektion etwa von sogenannten «Freundschaftsanfragen» bei Facebook, wird deshalb motiviert, weil Nutzer mit genau abschätzbaren, generalisierten Kommunikationsfolgen rechnen können. Angesichts unabwägbarer Kommunikationsfolgen, nicht abschätzbaren Peinlichkeiten, sind derartig umstandslose, direkte Anfragen Face-to-Face sonst allenfalls bei Kindern, die die Konsequenzen ihres kommunikativen Handelns eben noch erlernen müssen, möglich. Wobei selbst hier potentielle Peinlichkeit durch Schriftlichkeit, bzw. einen humoristischen Kontext, abgeschwächt wird: «Willst du mit mir gehen? - Ja/Nein (Bitte ankreuzen).»

Ebenso wie Schrift und Buchdruck nicht lediglich technische Hilfsmittel darstellen, etwa um mündliche Rede ortsun-

28 Dabei ist nicht von einer kongruenten technischen und symbolischen Generalisierung auszugehen, wodurch sich Möglichkeiten der Korruption ergeben. So lässt sich durch technische Generalisierung mittels sozialer Medien Liebe oder allenfalls Freundschaft in ihrer symbolischen Generalisierung - korrumpieren. Eine Möglichkeit, die bei der Rekrutierung von Frauen (oft jungen Mädchen) für den sogenannten «Islamischen Staat» (IS) genutzt wird (vgl. etwa Black, 2015; Nitsch, 2018).

29 Vgl. zu diesen Zahlen allfacebook.de: https:// allfacebook.de/toll/state-of-facebook. abhängig zu Verfügung zu stellen, sondern zu tiefgreifenden gesellschaftlichen Veränderungen geführt haben, lässt sich Adresslistenkommunikation nicht lediglich als Technik verstehen, mit der etwa bloss die Möglichkeiten der etablierten symbolisch generalisierten Kommunikationsmedien (etwa Geld, Macht, Wahrheit, Liebe) effizienter ausgestaltet würden. Auch wenn aufgrund der Neuheit dieser Formen von Kommunikation Folgen kaum abzuschätzen sind, kann mit gravierenden Konsequenzen gerechnet werden. So zeichnet sich mittlerweile empirisch ab, dass zukünftig reputationsorientierte Formen von Kommunikation an Bedeutung gewinnen (vgl. nachfolgend auch Abschnitt III.) Dies liegt daran, dass sich durch Adresslistenkommunikation die Adressierung von Kommunikation an eine Vielzahl von Personen normalisiert; gewissermassen die Adressierung an Publikum privatisiert, und nicht mehr nur in Ausnahmesituationen (wie etwa im Theater, bei Vorträgen, oder z. B. mittels verlagsunterstützter Publikationen) praktiziert wird. ${ }^{30}$

Adresslisten lassen sich demnach als «Zwitter» von Verbreitungsmedien und symbolisch generalisierten Kommunikationsmedien verstehen. Es geht hier darum, individuelle Erreichbarkeit mit Erfolg zu versehen, wahrscheinlich zu machen. Es ist diese Zwitterform, die es zulässt, dass hinsichtlich des Erfolgs von adresslistenbasierter Kommunikation unterschiedliche Schwerpunkte gesetzt werden können. So kann einerseits eine möglichst breitgestreute individuelle Erreichbarkeit als Erfolg im Vordergrund stehen (etwa bei sozialen Medien wie «Twitter» oder «Instagram»); andererseits mag die Etablierung einer tatsächlich einzigartigen individuellen Erreichbarkeit im Sinne

30 Beobachten lässt sich z. B., dass China plant, Reputation anhand eines «Punktesystems» zur Überwachung einzusetzen (vgl. «China plant die totale Überwachung», faz.net, 22.11.2017). Oder wie sich durch sogenannte "Influencer" Reputation wirtschaftlich ausnutzen lässt (vgl. etwa Kilian, 2017). Hinsichtlich allfälliger Gefahren zunehmender reputationsorientierter Kommunikation Räwel (2017). 
von höchstpersönlicher Kommunikation (Liebesbeziehungen) als Erfolg angestrebt werden (dann in Orientierung an Adresslisten wie etwa «Parship» oder «ElitePartner»).

Festzuhalten ist, dass die Nutzung aller Erfolgsmedien, sei es nun Macht, Geld, Liebe, oder Wahrheit, nicht voraussetzungslos erfolgen kann. Um Erfolgsmedien grundsätzlich erfolgreich nutzen zu können, ist nämlich schon vorweg ein erfolgreiches Erwartungsmanagement vorausgesetzt. So muss vorweg klar sein, dass es auf der Suche nach wissenschaftlicher Wahrheit sinnvoll ist, z. B. Universitäten aufzusuchen, nicht etwa Kirchen; dass man für die Möglichkeit des wahrscheinlichen Eigentumsübergangs mit Hilfe des Geldes (etwa von Büromaterial), dafür geeignete Geschäfte, und nicht etwa Amtsstuben aufsucht. Auch sollte klar sein, dass für die Ausübung von Macht etwa Kasernen geeignete Institutionen sind; hingegen die Anwendung von Macht beim Casting von Schauspielerinnen, um sexuelle Dienstleistungen zu erzwingen (Harvey Weinstein), tunlichst $\mathrm{zu}$ unterlassen ist. Ebenso muss vorweg klar sein, dass es für die Anbahnung, etwa von (Liebes-)Beziehungen oder Reisepartnerschaften, mehr oder weniger geeignete Adresslisten gibt. ${ }^{31}$

\section{Fallbeispiel: Die Anbahnung von (romantischer) Liebe}

\section{Hoffnungen}

Zugleich mit den Folgen gesellschaftlicher Phänomene geraten die Voraussetzungen dieser Folgen in den Blick. Untersuchte Kommunikation ist gerade aufgrund des Erfolgs von adresslistenbasierter Kommunikation von Interesse; also auch aufgrund der spezifischen Voraussetzungen, die zu

31 Es ist eine Forschungsfrage, ob ausreichend ist, die Aneignung dieser Erwartungserwartungen generalisierend der «Sozialisation» oder «Lebenspraxis» zuzurechnen. $\mathrm{Zu}$ vermuten ist, dass allenfalls an das Bildungsbzw. Erziehungssystem erhöhte Ansprüche gestellt werden, ein gesamtgesellschaftlich adäquates Erwartungsmanagement herauszubilden. diesen Folgen geführt haben. ${ }^{32}$ Und von einem Erfolg, indiziert durch die Popularität von Singlebörsen, ist insbesondere im Dunstkreis von Kommunikation im Medium der Liebe auszugehen:

The Internet is the one social arena that is unambiguously gaining in importance over time as a place heterosexual couples meet. For couples who met in 1990 and before, the percentage who met online was essentially zero. Between 1995 and 2005, there was exponential growth in the proportion of respondents who met their partners online, reaching what appears to be a plateau at approximately $22 \%$. For heterosexual couples who met in 2009, the Internet was the third most likely way of meeting, after the intermediation of friends, and approximately tied with the bars, restaurants and other public places. With the rise of the Internet as a way couples meet in the past few years, and the concomitant recent decline in the central role of friends, it is possible that the Internet could eventually eclipse friends as the most influential way Americans meet their romantic partners. (Rosenfeld \& Reuben 2012, S. 531)

Um Folgen abzuschätzen, ist zunächst zu verdeutlichen, wie voraussetzungsvoll, wie unwahrscheinlich höchstpersönliche Kommunikation, Kommunikation im Medium der Liebe überhaupt ist:

Für das Medium Liebe liegt [das] Problem in der höchstpersönlichen Kommunikation selbst. Unter höchstpersönlicher Kommunikation wollen wir eine Kommunikation verstehen, mit der der Sprecher sich von anderen Individuen zu unterscheiden sucht. (...) Gibt

32 Was letztlich ein Hinweis darauf ist, dass es sich bei der Gesellschaft um ein autopoietisches System handelt (vgl. Luhmann, 1997). Kommunikation ist nur im Netzwerk von Operationen möglich, die gerade diese Operationen selbst wiederum ermöglichen. Isolierte, an Kausalität orientierte Betrachtungen von Folgen, bzw. Voraussetzungen von Kommunikation sind daher wenig sinnvoll. Folgen können vielmehr gerade deshalb folgenreich sein, weil sie ihre Voraussetzungen stabilisieren. Veränderungen erfolgen in autopoietischen Systemen nicht kausal, sondern evolutionär. 
sich der andere als weltkonstituierende Individualität, ist jeder, der angesprochen wird, in dieser Welt immer schon untergebracht und damit unausweichlich vor die Alternative gestellt, den egozentrischen Weltentwurf des anderen zu bestätigen oder abzulehnen. Diese Komplementärrolle des Weltbestätigers wird einem [in der Liebe] zugemutet, obwohl mit impliziert ist, dass dieser Weltentwurf einzigartig, also eigenartig, also nicht konsensfähig ist. Das heisst auch: es wird einem ein Bestätigungsverhalten zugemutet, das nach aussen nicht anschlussfähig ist, das man also anderswo nicht vertreten kann. (...) Nicht Totalität, sondern Universalität des Bezuges wird erwartet im Sinne einer laufenden Mitbeachtung des Partners in allen Lebenslagen; man könnte auch sagen: einer laufenden Mitanreicherung des Informationsgehaltes aller Kommunikation durch den «für ihn» Aspekt. (Luhmann, 1982, S. 24-25, Hervorhebung durch den Autor) ${ }^{33}$

33 Wir können nur andeuten, dass dieses unwahrscheinliche, also extrem voraussetzungsvolle Medium wiederum von unwahrscheinlichen, nur durch soziale Evolution ermöglichten Voraussetzungen abhängig ist. So ist Liebe, zumal romantische Liebe, von der Ausdifferenzierung von Individualität abhängig, die wiederum erst durch funktionale Differenzierung der Gesellschaft ermöglicht wurde. Erst in der wesentlich durch die Erfindung des Buchdrucks ermöglichten Abkehr von gesellschaftlicher Stratifikation, die eher fremdreferentielle, also an Idealen orientierte Formen der - dann passionierten - Liebe plausibilisierte, konnten selbstreferentielle, nur an Individualität orientierte Formen der Liebe entwickelt werden, konnte Liebe in einem Kommunikationsmedium, konnte die Codierung von Liebe ausdifferenziert werden. So wurde erst in der romantischen Liebe, in Bezug auf die als sinnsteigernd, welterzeugend gedachte Relevanz von Individuen, ermöglicht, zu plausibilisieren, wenngleich sicherlich auch nicht erfolgreich zu realisieren, traditionell als flüchtig, fragil, paradox, ja unmöglich erscheinende Liebe widersinnig mit der Stabilität der Institution Ehe kurzzuschliessen. Uns bleibt in diesem Zusammenhang nur übrig, auf Luhmanns (1982) detaillierten Nachvollzug der Semantik der Liebe (deren Ideenevolution) zu verweisen.
Angesichts der extrem diffizilen Problemlage der Kommunikation im Medium der Liebe, wird deutlich, warum gerade in Adresslisten grosse Hoffnungen gesetzt werden. Angesichts der Normalität von unpersönlicher Kommunikation unserer hochgradig ausdifferenzierten Gesellschaft, zeigt sich, «dass sowohl im Erleben als auch im Handeln Schwierigkeiten des Anfangens auftreten, weil man in Situationen, die primär durch unpersönliche Erwartungen geordnet sind, ein Interesse am Persönlichen sehen und zum Ausdruck bringen muss, ohne dafür über gesellschaftlich geprägte Anlaufformen (Galanterie) zu verfügen.» (Luhmann, 1982, S. 205, Hervorhebung durch den Autor) Wenn überhaupt Kommunikation im Medium der Adresslisten einer klarer Erfolg zugesprochen werden kann, dann sicherlich hier. Wobei in diesem Abschnitt spezifisch von Adresslisten die Rede ist, die die Etablierung von langfristigen «Beziehungen» unterstützen, Adresslisten, wie etwa Parship, ElitePartner, eDarling usw. Dabei ist natürlich keineswegs ausgeschlossen, dass sich nicht auch bei anderen Formen der (Adresslisten-)Kommunikation (langfristige) (Liebes-)Beziehungen anbahnen können.

Technik liefert mittlerweile Chancen der Problemlösung, die weit über die Möglichkeiten von Galanterie (Luhmann, 1982, S. 97 ff.) der höfischen Gesellschaft hinausgehen. Galanterie ermöglichte in Oberschichten, Geselligkeit zum Trotz, intime Kommunikation. So gesehen stellen Adresslistenmedien (Singlebörsen) sozusagen ein funktionales Äquivalent der "Gesellschaft» in stratifizierten (höfischen) Gesellschaftsformen dar; in Zeiten also, in denen noch vorstellbar war, dass gesellige Interaktion «die Gesellschaft» repräsentieren konnte (vgl. Kieserling, 1999, S. 407 ff). Auch Angehörige von Oberschichten in stratifizierten Gesellschaften charakterisierten sich ja nicht durch ihre Individualität, sondern durch ihren Rang, bzw. ihre Distinktion von anderen (ebenso überindividuellen) Schichten. In diesem Sinne stellte die "Gesellschaft» in der geselligen Interaktion von Oberschichten (vgl. Luhmann, 1980) ein Kontaktmedium dar. 
Die Orientierung von Kommunikation im Medium der Liebe ausschliesslich an Sach- und Zeitdimension ist zentral. Die Sozialdimension, die Orientierung von Kommunikation an spezifischen, aber überindividuell Geltung beanspruchenden Rollen (soziale Generalisierung) muss aussen vor bleiben:

Über alle Nuancen von Aufrichtigkeit und Unaufrichtigkeit [im Handeln] hinweg und trotz häufiger Unentscheidbarkeit genau dieser Frage des Aufrichtigseins ist der $\mathrm{Zu}$ sammenhang von Handlung und individuellem Selbstsein der Schlüssel des Generalisierungsproblems - und eben deshalb kann nur sachliche und zeitliche, nicht aber soziale (für jedermann geltende) Generalisierung in Betracht kommen. (...) Man muss die eigene Identität als Garant für Dauer nämlich nicht statisch, sondern dynamisch einsetzen; nicht als so-wie-sie-immer-ist, sondern als an-derLiebe-wachsend. Die Berufung auf die eigene Identität macht ja zunächst gerade Unabhängigkeit von den Umständen und Unabhängigkeit von den Einflüssen anderer deutlich. Diese Sinnverweisung muss gelöscht bzw. ersetzt werden durch das Konzept der Identität-in-Transformation. Und dazu gehört, dass man gerade dem, den man liebt (und der sich selbst als dauerhaft weiss), bescheinigt, dass man durch ihn und durch die Liebe zu ihm das eigene Ich entfaltet. Identität muss also als Stabilitäts- und als Steigerungsbegriff zugleich gehandhabt werden. (Luhmann, 1982, S. $44-45)^{34}$

34 Insofern ist verfehlt, wenn Illouz (2011, S. $308 \mathrm{ff}$.) davon ausgeht, dass der Feminismus in seinem durchaus erfolgreichen Bestreben nach Gleichheit, damit auch einer Wahlmöglichkeiten fördernden Vergleichbarkeit zwischen den Geschlechtern, in der Orientierung von Beziehungen an «Äquivalenzprinzipien» (Illouz, 2011, S. $316 \mathrm{ff}$.), gewissermassen überzogen hat. Entgegen ihren eigenen Intentionen verdeutlicht Illouz (2013) anhand ihrer Analyse des weltweiten Bestsellers "50 Shades of Grey" nämlich, dass Liebe sich an «Identität-in-Transformation" steigert. Die Romantrilogie führt vor, wie sich Liebe gerade dadurch entwickelt, dass sie sich zunächst stereotyper Rollenbilder (junge, hübsche Literaturstudentin, bzw. attraktiver, mysteriöser Milliardär) entledigt
Kommunikation im Medium der Adresslisten, Kommunikation unter abwesenden Anwesenden erzwingt, dass Interaktionssysteme (Chatpartner) zunächst durch zeitliche und sachliche Sinndimension enttautologisiert werden (vgl. Abschnitt I.3.). Die zumindest anfängliche Abwesenheit von Wahrnehmung der gewissermassen invariablen Physis erleichtert nicht nur die «Löschung» sozialer Generalisierung, sondern unterstützt vielmehr, indem körperliche Wahrnehmung sukzessive zugelassen wird, das Konzept einer «Identität-in-Transformation». Identität wird hier gerade in Abkehr von etwa an Rollen orientierter üblicher unpersönlicher Kommunikation nicht vorausgesetzt, sondern muss in der Beschränkung auf zunächst schriftliche Kommunikation, in Orientierung an weitgehend anonymen Nutzerprofilen, erst selbstreferentiell konstruiert werden. Das dynamische Einsetzen von Identität, eine Berücksichtigung des Konzepts einer «Identität-in-Transformation», wird also durch die Nutzung des Adresslistenmediums selbst erzwungen. In Adresslistenmedien erfolgt sowohl eine Stabilisierung, wie auch Steigerung von Identität - etwa von Chats zu Telefonaten, von Bildtelefonaten zu Face-to-Face Kommunikation - auf gleichsam natürliche Weise.

Adresslistenkommunikation ermöglicht zudem an frühe Formen der Liebeskommunikation anzuknüpfen. Im 17. Jahrhundert wurde erstmals gesellschaftlich damit experimentiert, Inter-

und vielmehr Individualität konstruiert. Hier Hoffnungen auf die Sozialdimension zu setzen (Mehr Hierarchien zulassen? Dominanz in Rollen ausdifferenzieren?), also gerade von Individualität abzusehen, ist in der Liebe fehlgeleitet. Was nicht heissen soll, dass sich hier nicht in anderen Hinsichten Lösungsmöglichkeiten ergeben können - etwa: «Das Problem mit der Gleichheit oder «Fick mich endlich, verdammt noch mal!» (Illouz, 2013, S. 56) Wobei Liebe nicht mit Sexualität verwechselt werden sollte. Allerdings soll hier auch nicht bestritten werden, dass sich aus einem zunächst lediglich an Sexualität orientierten "Kontakt» (wie bei "50 Shades of Grey” vorgeführt) eine Liebesbeziehung entwickeln kann. 
aktionssysteme «freizugeben», also nicht anhand der Sozialdimension (orientiert etwa an gesellschaftlichen Schichten) zu enttautologisieren. Die Komplexität dieser sich neu ergebenden Freiheit - das Problem der doppelten Kontingenz wurde hier offensichtlich akut - wurde durch imaginative Idealisierung des Subjekts der Liebe reduziert:

In der Imagination verfügt man über die Freiheit des anderen, verschmilzt sie mit den eigenen Wünschen, übergreift die doppelte Kontingenz auf der Metaebene, die dem eigenen und dem anderen Ego das zuweist, was das eigene Ego für beide projiziert. Aber wie kommt es zur Imagination, wie schafft sie sich den Raum, und vor allem: wie schafft sie sich Zeit? Die Antwort liegt (...) in der Zentrierung auf die letzte Gunst - und in ihrem Aufschub. Die Idealfigur wird durch Temporalisierung der Liebessemantik abgelöst. (Luhmann, 1982, S. 62)

Auch Imagination leistet Adresslistenkommunikation gewissermassen auf natürliche Weise. Die Wahrnehmung der Physis ist hier (zunächst) extrem eingeschränkt, was Imagination zugutekommt. Die körperliche Abwesenheit erzwingt bzw. plausibilisiert den Aufschub der «letzten Gunst». Der in Chats abwesender Anwesender hochfrequente Wechsel von Beobachter- und Handlungsperspektiven, die hier mögliche kontrollierte Verdichtung von Interaktion, leistet einer Idealisierung Vorschub, privilegiert «Identität-in-Transformation", ermöglicht, dass Liebe an dieser Identität wachsen kann, leistet einem Prozess der Ausdifferenzierung komplexer (welterzeugender) und allenfalls idealer Individualität Vorschub.

Insofern lässt sich beim Medium der Adresslisten von geradezu idealen (Ausgangs-)Bedingungen für Initiierung und Konstitution von Kommunikation im Medium der Liebe ausgehen. Das Problem der doppelten Kontingenz muss - zunächst aus rein technischen Gründen - nach Massgabe von Sach- und Zeitdimension gehandhabt werden. Beim Zugriff auf Adresslisten ist von einem symmetrischen Sozialverhältnis der liebesuchenden Nut- zer auszugehen. Durch die zumindest zunächst gegebene Einschränkung der physischen Wahrnehmung, ist die Möglichkeit der Asymmetrisierung von interaktioneller Selbstreferenz durch die Sozialdimension, stark eingeschränkt. Identität muss (zunächst durch Chats, durch E-Mails usw.) vielmehr kommunikativ-reflexiv (erleichtert durch den einfachen Zugriff auf die Systemgeschichte, vgl. oben Abschnitt I.3.2.) ausgehandelt werden und kann so - als «Identität-in-Transformation» - gesteigert bzw. imaginiert (idealisiert) werden. Es kann davon ausgegangen werden, dass sich nicht zuletzt aufgrund dieser idealen (Idealisierung und Imagination Vorschub leistenden) Bedingungen, der erstaunliche Erfolg von Singlebörsen erklärt.

\section{Enttäuschungen}

Anders als Handlungstheorie, geht die Systemtheorie nicht von einer Kontinuität sichernden Stabilität handelnder Subjekte bei der Konstitution von Interaktionssystemen aus. Interaktionssysteme verstehen sich vielmehr als die flüchtigsten, fragilsten Systemformen der Gesellschaft. ${ }^{35}$ Der Übergang von Interaktionssystemen abwesender Anwesender (Chatpartner) zur Interaktionssystemen Anwesender (Face-to-Face Kommunikation) verläuft demnach diskontinuierlich. Diese Interaktionssysteme sind ganz unterschiedlich disponiert. Es werden grundlegend neue Interaktionssysteme etabliert. Kommunikation erfolgt nunmehr mündlich und der Körper ist als zusätzlicher Faktor der Umwelt eines Interaktionssystems nunmehr Anwesender zu berücksichtigen. ${ }^{36}$

35 Vgl. Kieserling (1999). Allenfalls lässt sich bei der Kommunikation auch abwesend Anwesender im Medium der Adresslisten, durch ständiges "online" sein, der Eindruck von Kontinuität erzeugen. Es kann davon ausgegangen werden, dass auch diese Wahrnehmung den Hoffnungen potentiell Liebender im Medium der Adresslisten Auftrieb verleiht; immerhin ist «totale» Kommunikation charakteristisch für Liebende (Luhmann, 1982, S. 24).

36 Wir müssen genauer sagen: der Körper ist nunmehr informativ zu berücksichtigen. In der Kommunikation abwesender Anwe- 
Gleichwohl natürlich bisherige Interaktionsgeschichte (als abwesende Anwesende) der Enttautologisierung doppelter Kontingenz des sich neu etablierenden Interaktionssystems der nunmehr Anwesenden dienlich ist, ist festhalten, dass die bisherige Systemgeschichte, etwa bereits erfolgte Kommunikation über spezifische Themen, vor allem aber bewusste Vorstellungen (Imaginationen) hinsichtlich der Individualität der nunmehr Anwesenden, der Umwelt des sich neu konstituierenden Interaktionssystems zugehörig ist. Die bisherige Systemgeschichte, die in der Kommunikation abwesender Anwesender bewusst-imaginativ konstruierte Individualität wirkt nicht determinierend, sondern irritierend auf das neu konstituierte Interaktionssystem nunmehr Anwesender. Individualität ist hier also, zumal an diesen Irritationen, neu zu konstruieren.

Mit Enttäuschung ist also, besonders bei der ohnehin extrem anforderungsreichen Kommunikation im Medium der Liebe, zu rechnen. ${ }^{37}$ Die sich durch not-

sender ist diese Informationsquelle weitgehend ausgeschaltet. Nämlich dadurch, dass Körper in der Benutzung der technischen Infrastruktur weitgehend «unterschiedslos» benutzt werden, in einer Unterschiedlichkeit also, die eben keinen informativen Unterschied ausmacht (vgl. zu diesem Informationsbegriff Bateson, 1972, S. 315). Körperbezogene Information (etwa Bilder und Emoticons) werden allenfalls kontrolliert zugänglich gemacht.

37 Aktuell diesen Enttäuschungen plausibel Ausdruck verleihend: Illouz (2011). Die Popularität, gewissermassen die Plausibilität der Plausibilität dieser Enttäuschungen, zeigt sich durch den Status dieses Sachbuchs als Bestseller. Festzustellen ist allerdings, dass hier ein Teil der Enttäuschungen nicht der Form der Kommunikation der Liebe, sondern der theoretischen Disposition dieses Sachbuchs zuzurechnen ist. Wird Liebe paradigmatisch (hier etwa mit Bezug auf die Romane von Jane Austen) auf den keineswegs selbstverständlichen Kurzschluss von romantischer Liebe und Ehe bezogen, kann, gerade angesichts moderner Wahlmöglichkeiten, eine Verfallsgeschichte von (Ehe-) Versprechen konstruiert werden (ebd., S. 186). Für die Moderne zeigt sich dann Bindungsangst geradezu als charakteristisch wendig neu zu konstituierende Interaktionssysteme ergebende Diskontinuität von Individualität - und eben nicht ihre Handhabung als «Stabilitäts- und Steigerungsbegriff» (Luhmann, 1982, S. 45) -, kann für bereits abwesend anwesend Liebende einem Drama gleichkommen. Zwar ist nicht ausgeschlossen, dass Kommunikation an bereits etablierte Individualität anschliessen kann, oder dass sich neu konstituierende Individualität auch mit sich erneut einstellender - Liebe kompatibel ist. Die hochgradig spezifischen Anforderungen der Liebe machen dies jedoch unwahrscheinlich. Zu berücksichtigen ist nämlich, dass Liebende, dadurch, dass sie den Eindruck haben, dass selbst der Umweltbezug, der individuelle Weltbezug des je Anderen (als Erlebende) erfolgreich, also bestätigend zum Ausgangspunkt ihres Handelns wurde, notwendig den Eindruck haben müssen, den anderen bereits bestens zu verstehen, bereits zu kennen und demnach von hochgradig differenzierten, detaillierten Vorstellungen von der Individualität des je anderen ausgehen. Gerade diese dezidierten Vorstellungen machen Enttäuschungen bei erstmaligen Treffen Face-to-Face - bei denen Individualität notwendig diskontinuiert wird - hochwahrscheinlich.

Diesem Problem mag mit gegensätzlichen Strategien entgegnet werden. Einerseits kann auf allzu intensive, erwartungsgenerierende Kommunikation abwesender Anwesender verzichtet werden (etwa in Orientierung an Adresslisten wie «Tinder $»^{38}$ ). Verzichtet wird damit

(ebd., 115ff). Diese Perspektive verfehlt allerdings, dass traditionell gerade die Instabilität, die Vergänglichkeit dem Kommunikationsmedium Liebe charakteristisch ist. Mit Blick auf Liebe als Passion gilt daher: «Nichts wäre abwegiger als bei Liebe an Ehe zu denken.» (Luhmann, 1982, S. 89) In diesem Zusammenhang ist allerdings generell von einer Enttäuschungsanfälligkeit der Handlungstheorie auszugehen, muss diese doch von der Kontinuität handelnder Subjekte ausgehen, die eine interaktionssystemübergreifende Erwartungssicherheit erwarten lässt.

38 Dabei soll in diesem Zusammenhang nicht behauptet werden, dass es bei Adresslisten 
aber auch auf das allenfalls das neue Interaktionssystem wirksam enttautologisierende Potential, das sich aus der Systemgeschichte, bereits kommunizierten Themen, Vorstellungen von Individualität (sogar allenfalls enttäuschten) ergibt, das eine im Entstehen begriffene «Beziehung» stabilisieren kann. Andererseits mag erfolgversprechend sein, so lange wie möglich ein Treffen (Face-to-Face Kommunikation) hinauszuzögern (was die Wahrscheinlichkeit von Enttäuschungen erhöht), oder eben ganz zu vermeiden, um die Liebe begründende Individualität der abwesenden Anwesenden nicht zu gefährden. ${ }^{39}$ Sich also mit platonischer Liebe $\mathrm{zu}$ begnügen. Ist dies aber Liebe, wenn vom Körper (von Sexualität) als essentiellem Bestandteil von Individualität abgesehen werden soll?

Es ist schwierig, festzustellen, ob die Kommunikation der Liebe in der Moderne mit mehr Enttäuschungen verbunden

wie «Tinder» vorrangig um die Anbahnung von Liebesbeziehungen geht. Nach eigenen, naturgemäss eher stichprobenartigen Recherchen wird "Tinder» auffallend geschlechtsspezifisch genutzt. Der epidemisch anzufindende Hinweis von Frauen (zumindest in der Alterskohorte 30+) in ihren Nutzerprofilen: Keine ONS! (One Night Stands), zeugt davon, dass es hier, ganz dem Geschlechterklischee entsprechend, Frauen oft auf die Etablierung von langfristigen (Liebes-)Beziehungen anlegen, während Männer bei «Tinder» oft eher nach sexuellen Abenteuern Ausschau halten.

39 Vgl. Illouz (2011, S. 420). Hier bezogen auf eine "virtuelle» Liebesbeziehung abwesender Anwesender. In drei Jahren kam es zu keinem Treffen. Ein nach zwei Jahren vereinbartes Treffen wurde kurzfristig abgesagt. Es ist in diesem Zusammenhang allerdings fehlgeleitet, von «fiktionalen Gefühlen» oder «interaktioneller fiktionaler Emotion» zu sprechen (ebd.). Das Gegenteil ist der Fall. Eher haben sich hier reale Gefühle von der Fiktionalität eines Treffens bedroht gesehen. Würde Handlungstheorie "den Menschen» in seiner Individualität ernst nehmen, wäre hier eher ein an Realität ausgerichtetes hohes Mass an Sensibilität zu konstatieren, als abschätzig von «fiktionalen Gefühlen» zu sprechen. Ein, liesse sich in Zusammenhang bemerken, durchaus liebenswerter Charakterzug. ist; zumal hier Vergleichskriterien fehlen. ${ }^{40}$ Wobei sich fragen lässt, ob die ohnehin extrem enttäuschungsanfällige Kommunikation im Medium der Liebe sinnvoller Weise überhaupt Steigerungsformen der Enttäuschung zulässt. Klar ist jedenfalls, dass handelnde Wahrnehmung von Ego in der Moderne zu berücksichtigen hat, dass, neben der "black box" des Bewusstseins, die "black box" der Adresslisten zunehmend integraler Bestandteil von Persönlichkeit, bzw. Individualität von Alter wird. ${ }^{41}$ Dies ist insofern nichts Neues, als ohnehin von sozialer Konstruiertheit von Individualität auszugehen ist.

Neu ist allerdings, dass Liebende nun gerade in der totalen Wahrnehmung, in der umfassenden Berücksichtigung des Weltbezugs anderer Individuen berücksichtigen und bestätigen müssen, dass in der Wahrnehmung dieser Individuen potentiell andere Individuen zugleich abwesend anwesend, und allenfalls auch von höchstpersönlichen Interesse sind. Mit Bezug auf (private) Adresslisten (in Smartphones) also gewissermassen konkreter und realistischer, als früher, als dies ledig-

40 Anders handlungstheoretisch orientierte Positionen. Hier ermöglicht der Bezug auf einen unveränderbaren (zeitstabilen?) «Kern» menschlicher Subjektivität die Vergleichbarkeit von Epochen in Relation auf Subjekte und so - die Moral von der Geschicht' - in einem generellen Sinne Personen «Verantwortlichkeit» und «Rechenschaft» zuzuweisen (vgl. Illouz, 2011, S. 437 ff.). Dies für Verhältnisse, in denen Systemtheorie die gesellschaftsstrukturell bedingte Disponiertheit von Individualität sieht und es allenfalls der Moral, bzw. dem Recht überlässt, Individuen Verantwortlichkeit oder Rechenschaft zuzuweisen. Aus systemtheoretischer Sicht ist festzustellen, dass Handlungstheorie, einmal mehr theoriebedingt, lediglich eine weitere Quelle für Enttäuschungen konstruiert.

41 In diesem Sinne ist nur konsequent, dass nach einem Urteil des Supreme Court in den USA, Smartphones «ein solch beherrschender und vereinnahmender Teil des täglichen Lebens [sind], dass der sprichwörtliche Besucher vom Mars sie für ein wichtiges Merkmal der menschlichen Anatomie halten könnte.» (Fast schon ein Körperteil. US-Urteil zu Smartphones, in: suedeutsche.de, 26.6.2014). 
lich bezogen auf die Sinnmöglichkeiten des intransparenten Bewusstseins der Fall war. Es ergibt sich das Paradox, dass gerade die totale Bezogenheit eines Liebenden auf ein Individuum die Mitberücksichtigung anderer Individuen erfordert. Es mag sein, dass dies Individuen überfordert. Zugleich den Bedarf nach höchstpersönlicher Kommunikation steigert und Strategien fördert, nicht zu starke Bindungen zuzulassen, um allfälligen Enttäuschungen zu entgegnen. ${ }^{42}$

\section{Reputation}

Es lässt sich fragen: Wenn bei Liebe, bezogen auf das Paradigma des Bewusstseins, die Orientierung eines Individuum an einem anderen Individuum zentral ist; wenn es hier um höchstpersönliche Kommunikation geht, darum, den anderen noch in seinem Umweltbezug, seiner Wahrnehmung, seinem individuellen, idiosynkratischen Weltbezug handelnd $\mathrm{zu}$ berücksichtigen und zu bestätigen ${ }^{43}$ - was könnte ein funktionales Äquivalent sein, bezogen auf das Paradigma der Adresslisten?

Man darf vermuten, dass bezogen auf Adresslisten - also mit Blick auf die "black box" von Individuen, aus der sich Sinnmöglichkeiten durch die intransparente Vielzahl von Kontakten ergibt - die Orientierung an generalisierten, allgemeinen Erwartungshaltungen zentral ist. Reputation kann, bezogen auf Adresslisten, als ein funktionales Äquivalent von «Liebe», bzw.

42 Extrem unterschiedliche Lebensformen, wie «Polyamory» oder «Hikikomori» (vgl. dazu die entsprechenden Wikipedia Artikel) könnten diese Tendenzen ausdrücken.

43 In der Liebe hat Ego in seinem Handeln auch noch die Wahrnehmung, den individuellen, idiosynkratischen Weltbezug von Alter zu bestätigen. Hass, könnte man von dieser Warte aus sagen, ist ganz ähnlich disponiert. Nur dass es hier nicht um Bestätigung, sondern um Ablehnung in der Komplettbetreuung von Individuen geht. So gesehen lässt sich verstehen, warum etwa im "Stalking" (sozusagen ein Atavismus passionierter Liebe), oder in sogenannten "On-Off Beziehungen», die eine Form relativ leicht in die andere umschlagen kann.
«Hass» gelten. Gemeint ist, dass hier nicht empathische, an Individuen ausgerichtete Kommunikation angestrebt wird, sondern Kommunikation an den Erwartungshaltungen eines Publikums ausgerichtet ist. Also Rollen in ihrer Generalisierung ausdifferenziert werden, denen eine Allgemeinheit, eine möglichst grosse Zahl an Personen, wenn vielleicht nicht «Liebe», so doch ein erhöhtes Mass an Aufmerksamkeit schenkt.

Reputation lässt sich demnach als ein Verfahren verstehen, durch welches versucht wird, das Problem der kontingenten Adressierung von Kommunikation in Adresslisten zu bewältigen. ${ }^{44}$ Reputation soll die Wahrscheinlichkeit erhöhen, dass Kommunikation in Adresslisten trotz einer unüberschaubaren Vielzahl von gleichartigen Elementen (potentiellen Adressaten) dennoch zustande kommt. In den sozialen Netzwerken lassen sich problemlos empirische Hinweise finden, dass Kommunikation im Medium der internetbasierten Adresslisten an Reputation ausgerichtet ist - etwa mit Blick auf die Fotosharing-Plattform Instagram:

Surft man bei Instagram, sticht die Professionalität vieler Teenagerprofile ins Auge. Perfektes Make-up, perfektes Styling, perfektes Licht. Die Inszenierungsspielregeln sind bekannt. Instagram ist eine Bühne. Es geht dabei nicht immer um Schönheit, aber sehr oft. Es existieren unzählige Apps, die den Fotos den professionellen Schliff verleihen. Im Grunde kann beinahe jeder, der den nötigen Aufwand betreibt, bei Instagram gut aussehen. Was unter dem Label Schnappschuss oder Selfie daherkommt, wirkt oft wie das Ergebnis eines durchgeplanten Fotoshootings. Die Bandbreite reicht von mädchenhaft verspielt bis eindeutig erotisch. Viel Haut bedeutet viele Likes. Likes sind die soziale Währung, ein

44 Die Einschränkung von Kontingenz - die graduelle Abschwächung von medialer Anonymität - bietet allerdings auch Möglichkeiten der Überwachung, wie das reputationsorientierte «Punktesystem» zeigt, das in China eingeführt werden soll. Für Gefahren, die mit zunehmend reputationsorientierter Kommunikation einhergehen Räwel (2017) (siehe auch Fussnote 30). 
permanent abrufbares Ranking, sichtbar für jeden. «Die mit besonders vielen Likes sind die (Fame-Leute», sagt Lara. «Die sind halt 〈Like-geil»». Sie wollen Applaus, jeder will Applaus, auch dieser Wettkampf ist Instagram Realität. Was für die «Fame-Leute» Segen ist, kann für jene, die ignoriert werden, Fluch sein. Unkommentierte Sichtbarkeit ist Unsichtbarkeit. (FAZ, Wer nicht auf dieser Bühne ist, ist raus, 17.1.2015, S. 13)

"Likes" ${ }^{45}$ können als Reputation indizierendes Surrogat der Wahrnehmung der Wahrnehmung anderer gelten. Allerdings kommt es dabei eben nicht auf die Wahrnehmung der Wahrnehmung eines Individuums an, darauf, dass ein Individuum auch noch die Wahrnehmung eines anderen Individuums, dessen individuellen Weltbezug zur Maxime des eigenen, bestätigenden, also liebevollen Handelns macht. Entscheidend ist vielmehr die $A n$ zahl der "Likes," also die Würdigung (oder doch wenigstens Aufmerksamkeit) eines allgemein-populären "Anderen», die sich im Idealfall zu einer Art Berühmtheit ( $« \mathrm{Fa}-$ me-Leute») auswachsen kann, bzw. soll. Reputation, ein Ranking in Bezug auf ein Adresslistenmedium ist durch die schlichte Anzahl der "Likes" durchaus messbar. Dies aber gerade indem von der Individualität, der einzigartigen Welt von individuellen Personen abgesehen wird.

Dabei kommt in diesem Zusammenhang "Likes" (etwa Facebook, Instagram), bzw. zudem "Dislikes" (etwa bei Youtube), "ReTweets” (bei Twitter), «Favoriten» (etwa bei Flickr) usw., zudem die Funktion zu, sicherzustellen, dass es hier überhaupt zur Kommunikation, zu einer strikten Koppelung im Medium kommt. Denn auch wenn an hunderte oder gar Tausende «Freunde» (Facebook) oder "Follower" (etwa Twitter, Instagram, Youtube usw.) Mitteilungen

45 Neben einer Vielzahl von anderen Möglichkeiten, die Wahrnehmung von Wahrnehmungen (allenfalls, in positiver Wendung: Anerkennung) zu indexieren. Etwa die Anzahl von "Followern" bei Twitter, Ranglisten, wie Verkaufszahlen oder Bestsellerlisten, Zitationsindizes, Länge von Publikationslisten, Anzahl von Kommentaren, Rang und Anzahl von Ergebnissen in Suchmaschinen usw. (etwa eine Nachricht, ein Bild, ein Video, ein link usw.) gesendet werden können, manifestiert sich eine strikte Koppelung (Kommunikation) doch erst dadurch, dass sichergestellt wird, dass - durch eine Anschlussmitteilung - eine Mitteilung von einer (latenten) Information (etwa: «Schau dir das an.», "Das ist interessant.») unterschieden und verstanden worden ist. Genau dies wird, auch wenn tausende von Personen adressiert wurden, durch zumindest ein "Like" / Dislike," "ReTweet" usw., geleistet.

Indiz dafür, dass Reputation oder zumindest Aufmerksamkeit, heute - man darf vermuten: geprägt durchaus durch die Einübung moderner Adresslistenkommunikation - oft nicht nur im persönlich-individuellen, sondern im unpersönlich-allgemeinen gesucht wird, mag die inflationäre Vielzahl der international im Fernsehen ausgestrahlten Castingshows $\operatorname{sein}^{46}$, die mutmasslich komplementär zu den deflationär eingeschätzten Persönlichkeitswahrnehmungen der Fernsehzuschauer stehen. Dem individuellen Eindruck also, dass die Möglichkeiten der persönlichen Wertschätzung von der Allgemeinheit unterschätzt werden. Ebenso mag an der Normalität, Häufigkeit und den Steigerungsraten von Schönheitsoperationen ein Bedarf an Reputation abgelesen werden. ${ }^{47}$

Spezifisch auf das symbolisch generalisierte Kommunikationsmedium Liebe bezogen, ist davon auszugehen, dass es hier nicht um Ersatz geht (obwohl auch dies in der modernen Einübung von Adresslistenkommunikation zunehmend der Fall sein kann), sondern eher um Er-

46 In Deutschland etwa «Deutschland sucht den Superstar», "The Voice of Germany," «Das Supertalent» usw. Vgl. für einen Überblick internationaler "Talent Shows" den Wikipedia Artikel "Castingshow."

47 So wurden im Jahr 1997 in den USA etwa 1.7 Mio. Schönheitsoperationen (Surgical and Nonsurgical Cosmetic Procedures) durchgeführt; im Jahr 2012 waren es etwa 10.1 Mio., im Jahr 2016 13.6 Mio. (Quelle: Cosmetic Surgery National Data Bank, Statistics, 2016: 4; vgl. http://www.surgery.org/ media/statistics). 
gänzung, die Erwartung, dass Reputation, gar Ruhm (unpersönliche Liebe) allenfalls der Konstitution höchstpersönlicher Kommunikation dienlich sein kann. Es spricht für die Unwahrscheinlichkeit von Kommunikation im Medium der Liebe ${ }^{48}$ und die Normalität und Dominanz unpersönlicher Kommunikation in der modernen, hochgradig funktional ausdifferenzierten Gesellschaft, dass die Nachfrage nach höchstpersönlicher Kommunikation (Liebe von Individuen) Ergänzung, wenn nicht sogar Ersatz, in einer anderen, extrem unwahrscheinlichen Form der Kommunikation sucht.

Deutlich wird allerdings durch technisch generalisierte Kommunikationsmedien, dass Reputation im Sinne ihres selektierenden und motivierenden Potentials nicht (mehr) nur als ein Mittel der Selbststeuerung einzelner Funktionssysteme $\mathrm{zu}$ verstehen ist. Etwa hinsichtlich Wissenschaft (vgl. Luhmann, 1991, insb. S. $237 \mathrm{ff}$.), oder in Bezug auf hier behandeltes Kommunikationsmedium Liebe. Reputation scheint zunehmend eine gesellschaftliche Relevanz zuzukommen. Eine nicht zuletzt durch die Infrastruktur des Internets sich mehr und mehr als «globales Dorf» (McLuhan \& Powers, 1989) wahrnehmende Gesellschaft macht offenkundig Instanzen notwendig, denen eine komplexitätsreduzierende "Vermittlungsrolle» zwischen den unterschiedlichen funktionalen Bereichen der Gesellschaft zukommt. «Dank der Abkürzungen und Erleichterungen, die es ermöglicht, eignet

48 «Liebe lässt sich nicht kaufen» - erzwingen. Im Gegensatz zu Geld als prinzipiell jeder Person zugänglichem symbolisch generalisiertem Kommunikationsmedium, ist Liebe an die Zufälligkeiten und zeitlichen Instabilitäten von sehr spezifischen Interaktionssystemen gebunden. Während wirtschaftlich der Eigentumsübergang mit Hilfe von Geld gewissermassen durch Geschäfte direkt organisierbar ist, sind in Bezug auf die Liebe allenfalls deren Anfangsbedingungen (zuvorderst das Kennenlernen) organisierbar. Dauerhafter Erfolg in der Liebe ist so gesehen vermutlich, verglichen mit anderen Erfolgsmedien, wie Geld, Wahrheit oder Macht, am unwahrscheinlichsten zu erreichen. [das] Medium symptomatischer Reputation sich vortrefflich für Vermittlungsdienste. (...) Ausserdem verbindet Reputation die Wissenschaft [aber eben nicht nur die Wissenschaft, J.R.] mit ihrer [innergesellschaftlichen, J.R.] gesellschaftlichen Umwelt.» (Luhmann, 1991, S. 237)

Dem angesprochenen reputationsorientierten «Punktesystem», das China plant einzuführen (siehe Fussnote 30), kommt eben nicht nur die Funktion der Überwachung $\mathrm{zu}$, sondern wird auch Vermittlungsfunktion in komplexen, modernen gesellschaftlichen Verhältnissen zugetraut. Inwiefern sich damit Reputation auch korrumpierend auswirken kann, bleibt abzuwarten. So ist etwa fraglich, ob es bei der Rekrutierung von Arbeitnehmern für Unternehmen zielführend ist, für spezifische berufliche Tätigkeiten auch doch «private» reputationsindizierende Facebook- oder Twitter-Profile der Bewerber mit einzubeziehen. Oder, andererseits, ob sich nicht durch entsprechend «frisierte» Profile in sozialen Medien beruflicher Ausrichtung (etwa XING oder LinkedIn) Möglichkeiten der Täuschung eines zukünftigen Arbeitgebers ergeben. ${ }^{49}$ Dass aber hier grundsätzlich Bedarf an Instanzen der Vermittlung besteht, ist angesichts von zunehmend komplexer werdenden Verhältnissen - etwa einem nunmehr etablierten europäischen Arbeitsmarkt durchaus verständlich.

Niklas Luhmann muss unzweifelhaft Weitsicht zugesprochen werden, da er schon Anfang der 70ziger Jahre des letzten Jahrhunderts prophezeite: «Steigende Komplexität wird die Diskrepanz der Selektionsbedürfnisse und der Motivationsbedürfnisse verschärfen, und es lässt sich nicht ausschliessen, ja fast erwarten, dass im Laufe weiterer Entwicklung auch hier eine funktionale Differenzierung eintreten muss, die das multifunktionale Medium der Reputation sprengt.» (Luhmann,

49 Siehe zur Veranschaulichung dieser neuen Erfordernisse: "Ihre Online-Reputation als Faktor bei der Jobsuche», Personalberatung MichaelPage, 9.2.2017; https://www.michaelpage.de/advice/karriere-tipps/job-social-media/ihre-online-reputation-als-faktor-bei-der-jobsuche. 
1991; zuerst 1970, S. 244) Zu sehen ist zunächst eine «Sprengung» des «Mediums der Reputation» im Sinne der Hypertrophie ihrer Bedeutung und Multifunktionalität. Bestrebungen, wie diejenigen in China, lassen im Sinne Luhmanns vermuten, dass sich mit Hilfe von Technik beginnt ein auch symbolische Geltung beanspruchendes generalisiertes Kommunikationsmedium der Reputation auszudifferenzieren. Die in diesem Medium von Personen je erreichten «Reputationspunkte» würden einer sozialen «Währung» gleichkommen, die mit symbolischer Bedeutung aufgeladen wäre und erfolgreich «Türen öffnen» würde - oder eben nicht.

\section{Acknowledgements}

Ich danke den/der/dem anonymen Gutachter/inn/en für wichtige und hilfreiche Hinweise und Kritik.

\section{Literatur}

Baecker, D. (2012). Observing networks: A note on asymmetrical social forms. $\mathrm{Cy}$ bernetics \& Human Knowing 19(4), 9-25. doi:10.2139/ssrn.1974810.

Baecker, D. (2007a). Studien zur nächsten Gesellschaft. Frankfurt am Main: Suhrkamp.

Baecker, D. (2007b). Network society. In N. O. Lehmann, L. Qvortrup, \& B. K. Walter (Hrsg.), The concept of the network society: Post-ontological reflections (S. 95-112). Copenhagen: Samfundslitteratur.

Bateson, G. (1972). Steps to an ecology of Mind: Collected essays in anthropology, psychiatry, evolution, and epistemology. Chicago: University of Chicago Press.

Black, L. (2015). The Islamic state's use of online social media. Military Cyber Affairs, 1(1). doi:10.5038/2378-0789.1.1.1004

Bourdieu, P. (1982). Die feinen Unterschiede. Kritik der gesellschaftlichen Urteilskraft. Frankfurt am Main: Suhrkamp.

Cacioppo, J.T., Cacioppo, S., Gonzaga, G. C., Ogburn, E. L., \& VanderWeele, T.J. (2013). Marital satisfaction and break-ups differ across on-line and off-line meeting venues. Proceedings of the National Academy of Sciences of the United States of Ameria, 110(25), 10135-10140. doi:10.1073/ pnas. 1222447110 .

Frank-Job, B., Mehler, A., \& Sutter, T. (2013). Die Dynamik sozialer und sprachlicher Netzwerke. Konzepte, Methoden und empirische Untersuchungen an Beispielen des WWW. Wiesbaden: Springer VS.

Fuchs, P. (2007). Das Mass aller Dinge: Eine Abhandlung zur Metaphysik des Menschen. Weilerswist: Velbrück.

Fuhse, J. (2009). Die kommunikative Konstruktion von Akteuren in Netzwerken. Soziale Systeme, 15(2), 288-316.

Goffmann, E. (1959). The presentation of self in everyday life. New York: Doubleday.

Heider, F. (1926). Ding und Medium. Symposium, 1, 109-157.

Huber, H. D. (1991). Interview mit Niklas Luhmann. Texte zur Kunst, 1(4), 121-133. doi:10.11588/artdok.00005492.

Illouz, E. (2013). Die neue Liebesordnung. Frauen, Männer und Shades of Grey. Frankfurt am Main: Suhrkamp.

Illouz, E. (2011). Warum Liebe weh tut. Eine soziologische Erklärung. Frankfurt am Main: Suhrkamp.

Kieserling, A. (1999). Kommunikation unter Anwesenden. Studien über Interaktionssysteme. Frankfurt am Main: Suhrkamp.

Kilian, K. (2017). Influencer Marketing Markenerfolg mit reichweitenstarken prominenten Testimonials. transfer Werbeforschung \& Praxis, 63(2), 60-66.

Kron, T., Lasarczyk, C.W. G., \& Schimank, U. (2003). Doppelte Kontingenz und die Bedeutung von Netzwerken für Kommunikationssysteme. Ergebnisse einer Simulationsstudie. Zeitschrift für Soziologie, 32(5), 374-395.

Lee R., \&Wellman, B. (2012). Networked: The new social operating system. Cambridge, MA.: MIT Press.

Luhmann, N. (1997). Die Gesellschaft der Gesellschaft. Frankfurt am Main: Suhrkamp.

Luhmann, N. (1994). Die Wirtschaft der Gesellschaft. Frankfurt am Main: Suhrkamp.

Luhmann, N. (1992). Die Wissenschaft der Gesellschaft. Frankfurt am Main: Suhrkamp.

Luhmann, N. (1991 [1970]). Selbststeuerung der Wissenschaft. In Soziologische Aufklärung I. Aufsätze zur Theorie sozialer Systeme. Opladen: Westdeutscher Verlag. 
Luhmann, N. (1984). Soziale Systeme. Frankfurt am Main: Suhrkamp.

Luhmann, N. (1982). Liebe als Passion. Frankfurt am Main: Suhrkamp.

Luhmann, N. (1981). Kommunikation über Recht in Interaktionssystemen. In Ausdifferenzierung des Rechts. Beiträge zur Rechtssoziologie. Frankfurt am Main: Suhrkamp.

Luhmann, N. (1980). Interaktion in Oberschichten: zur Transformation ihrer Semantik im 17. und 18. Jahrhundert. In Gesellschaftsstruktur und Semantik, Bd.1 (S. 72-162). Frankfurt am Main: Suhrkamp. McLuhan, M., \& Powers, B. R. (1989). The global village: transformations in world life and media in the $21^{\text {st }}$ century. New York: Oxford University Press.

Nietzsche, F. (1954 [1878]). Menschliches, Allzumenschliches I. München: Hanser.

Nitsch, H. (2018). Soziale Medien und (De-) Radikalisierung. In Rüdiger, T. G. \& Bayer, P. (Hrsg.), Digitale Polizeiarbeit (S. 65-90). Wiesbaden: Springer.

Räwel, J. (2017). Erbarmungsloses Gaffen. Soziale Medien und Empathie, Telepolis, 25.12.2017 (https://www.heise.de/tp/features/Erbarmungsloses-Gaffen-3917368. html).

Rosenfeld, J. R., \& Reuben, J.T. (2012). Searching for a mate. The rise of the Internet as a social intermediary. American Sociological Review, 77(4), 523-547. doi:10.1177/0003122412448050.

Trier, M., Bobrik, A., \& Bartels, T. (2007). Towards understanding the dynamics of digital communication networks. Wirtschaftsinformatik Proceedings 2007, Paper 90.

White, H. C . (1992). Identity and control: A structural theory of social action. Princeton, NJ: Princeton University Press.

\section{Internetreferenzen - Webseiten, Zeitungsartikel}

«Fast schon ein Körperteil. US-Urteil zu Smartphones», suedeutsche.de, 26.6.2014; http://www.sueddeutsche.de/digital/ us-urteil-zu-smartphones-fast-schon-einkoerperteil-1.2018007.

Cosmetic Surgery National Data Bank, Statistics, 2016; unter: http://www.surgery.org/ media/statistics.
«Wer nicht auf dieser Bühne ist, ist raus», FAZ vom 17.1.2015, S. 13; http://www.faz.net/ aktuell/feuilleton/debatten/instagram-aesthetik-die-neue-buehne-der-jugend-13374648.html.

Data Science of the Facebook World, 24. April 2013 http://blog.stephenwolfram. com/2013/04/data-science-of-the-facebook-world/.

«Statistiken zum Online Dating», Statista, https://de.statista.com/themen/885/online-dating/.

«Statistiken zu Facebook», https://allfacebook. $\mathrm{de} /$ toll/state-of-facebook.

«China plant die totale Überwachung», faz. net; 22.11.2017; http://www.faz.net/ aktuell/wirtschaft/china-plant-mit-nationalem-punktesystem-die-totale-ueberwachung-15303648.html.

«Ihre Online-Reputation als Faktor bei der Jobsuche», Personalberatung MichaelPage, 9.2.2017; https://www.michaelpage.de/ advice/karriere-tipps/job-social-media/ ihre-online-reputation-als-faktor-bei-derjobsuche. 